\title{
An RNase III processed, antisense RNA pair regulates a Campylobacter jejuni colonization factor
}

Sarah L. Svensson ${ }^{1} \&$ Cynthia M. Sharma ${ }^{1 *}$

${ }^{1}$ Chair of Molecular Infection Biology II, Institute of Molecular Infection Biology, University of Würzburg, 97080 Würzburg, Germany

*To whom correspondence should be addressed.

Tel: +49-931-3182-560

Fax: +49-931-3182-578

E-Mail : cynthia.sharma@uni-wuerzburg.de

Short title: RNase III processing of a bacterial sRNA

Keywords: small regulatory RNA/sRNA/pathogenesis/post-transcriptional regulation/RNase III/Campylobacter jejuni 


\section{ABSTRACT}

Small RNAs (sRNAs) are emerging as important and diverse post-transcriptional gene expression regulators in bacterial stress responses and virulence. While originally identified mainly in intergenic regions, genome-wide approaches have revealed sRNAs encoded in diverse contexts, such as processed from parental transcripts by RNase E. Despite its well-known roles in rRNA processing, RNA decay, cleavage of sRNA-mRNA duplexes, the role of RNase III in sRNA biogenesis is less well understood. Here, we show that a pair of cis-encoded sRNAs (CJnc190 and CJnc180) are processed by RNase III in the foodborne pathogen Campylobacter jejuni. While CJnc180 processing requires CJnc190, RNase III cleaves an intramolecular duplex in CJnc190, independent of CJnc180. Moreover, we demonstrate that CJnc190 directly represses translation of the colonization factor PtmG by binding its G-rich ribosome binding site, and show that CJnc180 is a cis-acting antagonist of CJnc190, thereby indirectly affecting $p t m G$ regulation. Our results expand the diversity of known genomic locations of bacterial sRNA sponges and highlight a role for bacterial RNase III that parallels miRNA processing by related eukaryotic Dicer and Drosha. 


\section{INTRODUCTION}

Bacterial small, regulatory RNAs (sRNAs) are an important class of post-transcriptional gene expression regulators that control adaptation to changing environmental conditions or stresses (Storz et al, 2011), or can also regulate virulence genes in pathogens (Westermann, 2018; Svensson \& Sharma, 2016; Quereda \& Cossart, 2017). They are also intimately associated with RNA-binding proteins (RBPs), such as RNA chaperones, as well as ribonucleases that are required for their maturation, stability, degradation, and/or function (Holmqvist \& Vogel, 2018; Quendera et al, 2020). While most of the first identified and characterized sRNAs in bacterial genomes are expressed as stand-alone, intergenicallyencoded transcripts, genome-wide RNA-seq based approaches have identified sRNAs hidden in unexpected genomic contexts, including $5^{\prime} / 3^{\prime}$ untranslated regions (UTRs) and coding regions of mRNAs, or even in housekeeping RNAs (reviewed in (Adams \& Storz, 2020)). These include members of the expanding class of 3' UTR-derived sRNAs studied in Gammaproteobacteria (Miyakoshi et al, 2015a), which can be transcribed from an independent promoter such as E. coli MicL (Guo et al, 2014; Chao et al, 2012), or can be processed from mRNAs by the single-stranded RNA endonuclease RNase E (Chao \& Vogel, 2016; De Mets et al, 2019). Intergenically-encoded, stand-alone sRNAs can also require maturation by RNase E to increase their stability (Davis \& Waldor, 2007; Chae et al, 2011; Hör et al, 2020), expose their seed regions (Papenfort et al, 2009), or even create two sRNAs with distinct regulons (Papenfort et al, 2015; Fröhlich et al, 2016).

Despite progress in defining mechanisms and regulatory consequences of complex sRNA biogenesis pathways in model Gammaproteobacteria, less is known about how such sRNAs are generated in bacteria lacking RNase E. While RNase E is present in $\sim 20 \%$ of 
bacterial species (Hui et al, 2014), the double strand-specific RNase III is almost universally conserved in bacteria (Court et al, 2013). Bacterial RNase III is mainly known for its role in rRNA processing, maturation or decay of certain mRNAs, and cleavage of sRNA-mRNA duplexes (Court et al, 2013). While the RNase III domain-containing Dicer and Drosha play a central role in sRNA biogenesis in eukaryotes (Carthew \& Sontheimer, 2009), its role in sRNA biogenesis in bacteria is less clear. However, its expropriation by Type-II CRISPR/Cas systems for biogenesis of CRISPR RNAs (Deltcheva et al, 2011; Dugar et al, 2018), as well as genome-wide studies of the RNase III targetome that report sRNAs as potential targets in Gram-negative as well as Gram-positive species (Altuvia et al, 2018; Lioliou et al, 2012, 2013; Gordon et al, 2017; Le Rhun et al, 2017; Rath et al, 2017; Lybecker et al, 2014), indicate the potential for a broad role in sRNA processing in bacteria.

In addition to an expanding context of genomic regulatory RNA sources, there is also emerging evidence for high complexity in bacterial post-transcriptional networks involving not only cross-talk with transcriptional control, but also RNAs that can sequester and modulate RNA-binding proteins (Romeo \& Babitzke, 2018; Wassarman, 2018) or even regulate stability or function of other RNAs as so-called competing endogenous RNAs (ceRNAs), RNA decoys/predators, or sponge RNAs (Figueroa-Bossi \& Bossi, 2018; Grüll \& Massé, 2019; Kavita et al, 2018). Such RNA sponges can be derived from diverse cellular transcripts, including mRNAs (Miyakoshi et al, 2015b; Adams et al, 2021; Figueroa-Bossi et al, 2009) and tRNAs (Lalaouna et al, 2015), or can be stand-alone sRNAs encoded in the core genome or in prophages (Melamed et al, 2020; Tree et al, 2014; Bronesky et al, 2019). Despite reports of extensive antisense transcription in diverse bacteria and the demonstrated role of asRNAs in control of mRNA translation and stability (Thomason \& 
Storz, 2010), less is known about whether RNAs encoded in cis to other sRNAs can act also as RNA sponges or how they might affect the biogenesis, stability, or function of their antisense sRNA partners. The overlapping nature of these transcripts also means that RNase III might play a broader role in gene expression control via participation in RNA sponging.

The zoonotic food-borne pathogen Campylobacter jejuni is currently the leading cause of bacterial foodborne gastroenteritis worldwide and is also associated with serious post-infectious neuropathies such as Guillain-Barré syndrome (Burnham \& Hendrixson, 2018; Havelaar et al, 2015). So far, how C. jejuni regulates its gene expression to adapt to different environments is unclear, as its genome lacks homologs of certain global stress response regulators and encodes for only three sigma factors (Parkhill et al, 2000; Young et al, 2007). Our differential RNA-seq (dRNA-seq) based comparative transcriptome analysis of multiple $C$. jejuni strains revealed many conserved and strain-specific sRNAs and asRNAs (Dugar et al, 2013). However, the functions are largely unknown for most of these. Besides having a small number of transcriptional regulators, $C$. jejuni also lacks homologs of the global RNA chaperones Hfq and ProQ (Pernitzsch \& Sharma, 2012; Quendera et al, 2020), making it unclear how its sRNAs act. It is also largely unknown which RNases participate in sRNA biogenesis and function in C. jejuni. Although C. jejuni is a Gram-negative, Epsilonproteobacteria surprisingly encode an RNase repertoire more similar to Grampositives: for example, RNase Y and RNase J instead of RNase E (Pernitzsch \& Sharma, 2012; Parkhill et al, 2000; Tomb et al, 1997), making it unclear how biogenesis of processed sRNAs might occur. C. jejuni encodes an RNase III homolog (Haddad et al, 2013) 
which participates in the biogenesis of Type II-C CRISPR RNAs (Dugar et al, 2013, 2018). Beyond this and a role in rRNA biogenesis, the function of $C$. jejuni RNase III is unclear.

Here we have characterized the biogenesis and mode of action of a conserved, processed pair of $C$. jejuni cis-encoded, antisense RNAs, CJnc180/190. We had recently reported that deletion of these sRNAs affects $C$. jejuni virulence in a three-dimensional tissue-engineered model of the human intestine (Alzheimer et al, 2020). While this seemed to be at least in part mediated via repression of the flagellin modification factor PtmG, the underlying molecular mechanisms of this, the roles of the two sRNAs in PtmG regulation, as well as how they are processed remained unknown. Here, we demonstrate that both RNAs are processed by RNase III and that mature CJnc190 directly represses translation of $p t m G$ mRNA by base-pairing with a G-rich sequence over its RBS. Surprisingly, although both RNAs are expressed antisense to each other, suggesting co-processing by RNase III, only CJnc180 requires its antisense partner for maturation. Instead, CJnc190 is transcribed as longer precursors which can fold into extended stem-loop structures that are processed independently of CJnc180 by RNase III. Finally, we demonstrate that CJnc180, despite not being required for maturation of CJnc190, is a cis-acting antagonist of CJnc190.

Characterization of the complex CJnc190 biogenesis pathway points toward a broader role for RNase III in sRNA processing in bacteria than has been previously appreciated and reveals cis-encoded sRNA-sRNA targeting. 


\section{RESULTS}

\section{A product of the CJnc180/190 sRNA locus represses expression of ptmG}

Our comparative dRNA-seq study of multiple $C$. jejuni isolates revealed a conserved pair of antisense sRNAs, CJnc180 and CJnc190, which we had annotated as 99 and 216 nucleotides (nt), respectively, in strain NCTC11168 (Dugar et al, 2013) (Fig 1A). RNA-seq patterns as well as northern blot analysis suggested that both sRNAs might be processed from longer forms (Dugar et al, 2013). While CJnc180 was detected in wild-type (WT) as both a 90 nt "mature" 5' end-derived and a 160 nt longer putative precursor (pre-CJnc180) on northern blots, only a single CJnc190 species ( $70 \mathrm{nt})$ was detected, appearing to arise from the $3^{\prime}$ end of its annotated primary transcript (pre-CJnc190). Mature CJnc180 (hereafter, CJnc180) shows almost complete complementarity to mature CJnc190 (hereafter, CJnc190, Fig 1A).

We previously observed that deletion of CJnc180/190 affects C. jejuni adherence and internalization in our Caco-2 cell based tissue-engineered model of the human intestine (Alzheimer et al. 2020), suggesting CJnc180 and/or CJnc190 might regulate C. jejuni virulence genes. Comparison of the total protein profile of a $\Delta \mathrm{CJnc180/CJnc190} \mathrm{deletion}$ mutant $(\Delta 180 / 190)$ to that of the parental WT strain by SDS-PAGE revealed an approximately $45 \mathrm{kDa}$ band upregulated upon deletion of CJnc180/190 (Fig 1B). Complementation of $\Delta 180 / 190$ with a region spanning the Cj1650 and map intergenic region (C-180/190, dashed line, Fig 1A) at the unrelated $r d x A$ locus completely rescued expression of both sRNAs and restored repression of the $45 \mathrm{kDa}$ protein band (Fig 1B). 
Mass spectrometry analysis indicated that the upregulated protein band is the flagellin modification factor PtmG (Cj1324) (Fig S1; Table S1). The upregulated band was no longer observed in a $\Delta 180 / 190 \Delta p t m G$ double mutant, confirming it as PtmG (Fig 1B). Northern blot analysis further demonstrated that not only the protein level but also ptmG mRNA levels are also upregulated almost 10-fold in $\Delta 180 / 190$ vs. WT and are reduced (2fold) upon overexpression of CJnc180/190 by introduction of a second copy of CJnc180/190 into $r d x A$ of WT (OE-180/190). Overall, these data indicate that at least one of the two sRNAs of the CJnc180/190 locus is involved in repression of $p t m G$, encoding a colonization/infection-relevant flagellin modification factor (Howard et al, 2009;

Alzheimer et al, 2020).

\section{The mature CJnc190 sRNA is sufficient to repress ptmG}

To disentangle the roles of each sRNA in potential direct regulation of $p t m G$, we first defined the boundaries of each mature sRNA in WT. Primer extension mapped the $5^{\prime}$ end of CJnc180 in WT and C-180/190 to the nucleotide after its annotated TSS (Fig S2A). The 3' end of CJnc180 suggested by 3'RACE (rapid amplification of cDNA ends) was detected $\sim 88$ nt downstream of its TSS (Fig S2B), consistent with its observed northern blot length, although we also observed additional 3' ends for longer species ( 99 - $199 \mathrm{nt})$. The mature 5' end of CJnc190 was mapped to two nucleotides (A/G) 150 nt downstream of its annotated TSS and its $3^{\prime}$ end was consistent with its apparent length of $\sim 65-70$ nt on northern blots (Fig S2C, lanes 5/7 \& Fig S2D). Based on these results, we concluded that both sRNAs have slightly variable $3^{\prime}$ ends and that mature CJnc180 ( $\left.\sim 88 \mathrm{nt}\right)$ is derived 
from the $5^{\prime}$ end of pre-CJnc180, while mature CJnc190 ( $\left.\sim 69 \mathrm{nt}\right)$ lacks the $5^{\prime}$ end of its primary transcript.

Predicted secondary structures and potential $p t m G$ mRNA interactions of the mature sRNAs revealed a strong potential for a C/U-rich loop within CJnc190 to base-pair with the ribosome binding site (RBS) and start codon of $p t m G$ mRNA (Fig 1C), indicating potential translational repression. In contrast, a less stable interaction $(\Delta \mathrm{G}=-6.4 \mathrm{vs}$. -16.9 $\mathrm{kcal} / \mathrm{mol}$ for CJnc190:ptmG) was predicted between CJnc180 and the $3^{\prime}$ end of the $p t m G$ coding region, strongly suggesting that CJnc190, and not CJnc180, directly represses ptmG. To obtain experimental evidence for CJnc190 repression of $p t m G$, we constructed $\Delta 180 / 190$ complementation strains expressing either mature CJnc180 or CJnc190 from their annotated native promoters (P1) and measuring rescue of $p t m G$ repression (Fig 1D, left). Northern blot analysis confirmed expression of a CJnc180-rrnB terminator fusion (88 nt of mature CJnc180) with the expected size in the C-180(Proc) strain as well as expression of an sRNA of similar size to WT for strain C-190(Proc) via fusion of its mature 5' end to its annotated TSS (Fig S3A, lanes 4 \& 5). CJnc190 appeared slightly longer and more abundant in this context but this is likely due to small $3^{\prime}$ end differences as its $5^{\prime}$ end was confirmed to be the same as in WT by primer extension (Fig S2C, lane 8). Western blot analysis of a chromosomally epitope-tagged PtmG-3×FLAG fusion showed 1.5-fold upregulation upon deletion of CJnc180/190 and complementation to WT levels in C180/190 (Fig 1D, right). While C-180(Proc) did not show restored regulation of PtmG3×FLAG, the C-190(Proc) complementation strain showed PtmG-3×FLAG levels comparable to WT. A similar trend was seen for $p t m G$ mRNA levels (Fig S3A). These in vivo observations are consistent with the prediction that mature CJnc190, and not CJnc180, base 
pairs with the RBS of $p t m G$ to repress its translation. Moreover, a translational GFP reporter fusion of the 5'UTR and first 10 codons of ptmG (ptmG(10th)-GFP) was repressed by CJnc190 when transcribed from either its native promoter or the unrelated $\sigma^{28}(\mathrm{FliA})$ dependent flaA promoter, confirming that regulation is at the post-transcriptional level

(Fig S3B). Collectively, these observations showed that CJnc180 is dispensable and mature CJnc190 is sufficient for post-transcriptional repression of $p t m G$.

\section{CJnc190 represses ptmG translation by base-pairing with its G-rich RBS}

We next used in vitro and in vivo experiments, together with compensatory base-pair exchanges within the predicted CJnc190-ptmG duplex, to validate direct interaction (Fig

2A) and regulation of $p t m G$ by CJnc190. Gel mobility shift assays using in vitro-transcribed RNAs showed that processed CJnc190 binds the ptmG leader, and that a single C-to-G change in its C/U-rich loop (M1) is sufficient to almost completely abolish complex formation (Fig 2B). Similarly, a single point mutation (M1') in the $p t m G 5^{\prime} \mathrm{UTR}$ also disrupted interaction with CJnc190, and binding was restored when RNAs containing compensatory base exchanges were incubated together. Mature CJnc180 did not bind ptmG, in line with the weak predicted CJnc180:ptmG interaction. Reciprocal experiments with labeled CJnc190 (WT/M1) and unlabeled ptmG leader (WT/M1') further confirmed the interaction (Fig S4A). To confirm nucleotides participating in the CJnc190-ptmG interaction, we performed Inline probing with in vitro-transcribed RNAs. Increasing amounts of unlabeled $p t m G$ leader (WT) protected nucleotides in the C/U rich loop region of $5^{\prime}$ end labeled mature CJnc190 from cleavage, in agreement with the predicted interaction (Fig 2A \& 2C). The same molar ratio of $p t m G \mathrm{M} 1^{\prime}$ mutant leader showed less 
protection, indicating destabilization of the interaction. Reciprocal experiments with labeled WT ptmG leader and WT or M1 CJnc190 further confirmed the predicted interaction site on $p t m G$ (Fig S4B).

Base-pairing with a G-rich sequence at the $p t m G$ RBS suggested CJnc190 might act by repressing $p t m G$ translation. Indeed, the addition of increasing molar ratios $(1-, 10-, 50-$ fold; + to +++) of mature CJnc190 to an in vitro translation system with a ptmG-gfp translational reporter mRNA as template repressed GFP levels in a dose-dependent manner (Fig 2D). Consistent with the disrupted interaction, the M1 mutation in the CJnc190 loop partially restored translation of the reporter. The $\mathrm{M} 1^{\prime}$ mutation in the $p t m G$ reporter increased its translation compared to the WT template, independent of CJnc190 addition. However, inline probing experiments did not reveal marked differences in secondary structure for the native (non-GFP-fusion) WT and M1 ptmG leaders in vitro (Fig S4B). Nonetheless, while the addition of CJnc190 WT did not strongly affect translation of the mutant reporter, CJnc190 M1, carrying the compensatory exchange in its C/U-rich loop, strongly reduced GFP levels generated from the M1' reporter, indicating restored regulation (Fig 2D).

Finally, we confirmed that direct CJnc190:ptmG interaction mediates ptmG repression in vivo. Introduction of the $\mathrm{M}^{\prime}$ mutation in the $p t m G(10 \mathrm{th})-\mathrm{GFP}$ reporter fusion derepressed GFP levels, although not to those of an isogenic $\Delta 180 / 190$ strain (Fig 2E, left). To account for different levels of CJnc190 in the WT and C-190(Proc), we next compared regulation of the reporter by WT/M1 CJnc190 in the C-190(Proc) background. In line with a disrupted interaction, CJnc190 M1 did not repress the WT ptmG reporter to the same levels as CJnc190 WT (Fig 2E, right), even though WT/M1 sRNAs were similarly expressed 
(Fig S4C). Likewise, when WT CJnc190 was expressed with the $p t m G \mathrm{M} 1$ ' reporter, GFP levels were also higher compared to the WT sRNA/leader strain. Finally, in the strain with the compensatory mutations combined (M1/M1'), GFP levels were similar to the isogenic WT/WT (Fig 2E). Together, our in vitro and in vivo experiments demonstrate that CJnc190 represses $p t m G$ translation via base-pairing with its RBS.

\section{RNase III processes the CJnc180/190 sRNAs}

After validating direct interaction and post-transcriptional repression of $p t m G$ by CJnc190, we set out to gain insight into the biogenesis of the CJnc180/190 sRNA pair. Examination of potential processing defects in deletion mutants of non-essential RNases/RNA degradation enzymes revealed that most of the deletions had no major impact on processing of the two RNAs (Fig S5A). However, deletion of double-strand RNA specific RNase III ( $\Delta r n c)$ had a dramatic effect on both CJnc180 and CJnc190, abolishing accumulation of the mature sRNA species (Fig 3A). For CJnc180, the longer transcript of WT (pre-CJnc180, 160 nt) was still detected in $\Delta r n c$, but at higher levels. For CJnc190, the mature form of WT was completely absent in $\Delta r n c$ and instead five longer "pre-CJnc190" species ( 150-280 nt) were detected. All CJnc180 and CJnc190 unprocessed species were absent in a $\Delta 180 / 190 \Delta r n c$ double mutant, ruling out cross-hybridization.

We next asked if RNase III processing of CJnc190 affects ptmG regulation. Deletion of rnc increased PtmG-3×FLAG protein and mRNA levels to those similar to $\Delta 180 / 190$ (Fig 3B

\& Fig S5B), and CJnc180/190 deletion in $\Delta r n c$ did not increase levels further. In contrast to "native" CJnc190 (i.e., processed from precursors), the "mature" sRNA of the C-190(Proc) strain (transcribed directly from its mature $5^{\prime}$ end) was not markedly affected by rnc 
deletion (Fig S2B) and was still capable of $p t m G$ regulation in the $r n c$ deletion background

(Fig 3B). The mild increase in ptmG mRNA levels upon rnc deletion in C-190(Proc) suggests that RNase III could also play a minor role in cleavage of CJnc190:ptmG duplexes (Fig S5B). The combined levels of all CJnc190 species were 10 -fold lower in $\Delta r n c$ compared to WT (Fig 3A, compare lanes 1 and 4). Rifampicin stability assays showed that the CJnc180 precursor was stabilized in the $\Delta r n c$ mutant when compared to WT (Fig S5C), which further validates processing by RNase III. Moreover, while the half-life of mature CJnc190 in the WT strain was $>64 \mathrm{~min}, \mathrm{CJnc} 190$ precursors had half-lives of $2-4 \mathrm{~min}$ in the $\Delta r n c$ mutant. Taken together, these results indicate that RNase III processes both sRNAs and affects $p t m G$ regulation, likely by generating a more stable CJnc190 sRNA species.

\section{CJnc190 precursors are transcribed from two promoters and have diverse $3^{\prime}$ ends}

To further understand the unique RNase III-mediated processing of CJnc180 and CJnc190, we next characterized their precursors. Northern blot analysis with a probe binding the $3^{\prime}$ end of CJnc180 RNA-seq coverage showed that this end is removed by RNase III processing (Figs S6A \& 6B, probe 2). The 5' end, in contrast, was RNase III independent (Fig S2A). All CJnc190 precursors in $\Delta r n c$ hybridized to a probe binding upstream of the processed sRNA

(Figs S6A \& S6C, probe 4). In contrast, a second probe complementary to the far $5^{\prime}$ end (probe 5) detected only three of the five bands in $\Delta r n c$, suggesting that CJnc190 precursors differ in their 5' ends. Probes binding downstream of mature CJnc190 (probes 6 - 8) revealed that a longer $3^{\prime}$ end is present in most precursors. Therefore, we concluded that mature CJnc190 might be excised from the middle and/or 3' end of pre-CJnc190 transcripts expressed from multiple promoters. 
We next characterized the potential promoters driving expression of pre-CJnc180 and pre-CJnc190 to understand the source of the diverse pre-CJnc190 5' ends. While a single TSS (promoter P1) for CJnc190 was previously annotated in NCTC11168 based on dRNA-seq of WT (Dugar et al, 2013), alignments of the Cj1650-map intergenic region from C. jejuni, $C$. coli, and $C$. hepaticus strains revealed a conserved $\sigma^{70}$ near-consensus (TAAAAT) downstream of annotated P1 (Fig S7, promoter P2). To validate activity of the three putative CJnc180/190 promoters [annotated CJnc180(P1) and CJnc190(P1); predicted CJnc190(P2)] in vivo, we performed site-directed mutagenesis of their respective -10 boxes in the C-180/190 complementation construct (Fig 3C). First, the CJnc180(P1) promoter was disrupted (see Table S5 for mutations) to create strain C-190-only [CJnc190(P1) and CJnc190(P2) active]. To this allele, CJnc190(P1) or (P2) promoter inactivation mutations were added to create strains C-190(P2) only and C-190(P1) only, respectively. Finally, a strain with all three putative promoters mutated (C-3×mut) was also constructed.

Analysis of CJnc180 precursors in a $\Delta r n c$ background in the generated promoter mutant strains showed that disruption of the annotated CJnc180 promoter (strain C-190 only) fully abolished CJnc180 expression (Fig 3C), confirming its single TSS. CJnc180 promoter disruption reduced accumulation of some CJnc190 precursors, but did not affect their $5^{\prime}$ ends, suggesting transcriptional interference might impact $3^{\prime}$ ends. Next, we inspected pre-CJnc190 expression in strains with disruptions in either CJnc190(P1) or CJnc190(P2) added to the C-190 only construct [i.e., strains C-190(P2) and C-190(P1), respectively]. In strain C-190(P2) (CJnc180 and CJnc190 P1 promoters inactivated), the two longest CJnc190 species of $\Delta r n c$ were absent, and detection of a shorter CJnc190 transcript supported the presence of a second promoter (Fig 3C, lane 6). In the C-190(P1) 
strain, instead only a longer ( 280 nt) transcript was detected (lane 7). Finally, disruption of all three putative promoters (C-3×mut) completely abolished expression of all CJnc180 and CJnc190 transcripts (lane 8). Primer extension of the same strains validated the position of the CJnc190 P1 TSS, as well as a second CJnc190 5' end, whose position was consistent with the conserved P2 -10 box (Fig 3D \& Fig S7). Both $5^{\prime}$ ends were absent in the C-3×mut strain and no other $5^{\prime}$ ends were detected (Fig S8A).

We next used $3^{\prime}$ RACE in $\Delta r n c$ to identify precursor 3' ends (Fig S9). The mature (88 nt) 3' end of CJnc180 in WT was absent in $\Delta r n c$. Instead, we detected positions corresponding to RNAs of $\sim 104$ nt and $\sim 154$ nt (based on the TSS), consistent with northern blots (Fig 3A). RACE clone sequences from $\Delta r n c$ suggested that CJnc190 precursors might have one major and one minor $3^{\prime}$ end. Consistent with northern blot probing, these were longer than the mature sRNA. The linker-junction of a single $\Delta r n c$ RACE clone coincided with the mature CJnc190 3' end, which is followed by four T residues, suggesting SL2 could terminate transcription. Combining two $5^{\prime}$ and three $3^{\prime}$ end positions, we detected transcripts of lengths 279/248/213 nt and 216/186/151 nt (transcribed from P1 and P2, respectively), with the most abundant species ( $186 \mathrm{nt})$ likely arising from P2 with also a 3' extension (Fig S6 \& S9). Interestingly, this CJnc190 3' end position was immediately upstream of the CJnc180 promoter on the opposite strand (Fig S9), in line with our observation that abolishing CJnc180 expression reduced levels of some CJnc190 precursors with shorter 3' ends (Fig 3C). Overall, mapping of CJnc180 and CJnc190 precursors in $\Delta r n c$ suggested that mature CJnc180 is transcribed from a single promoter and derived from its precursor 5' end, while mature CJnc190 is generated from transcripts 
with different $3^{\prime}$ end lengths arising from two promoters, suggesting that CJnc190 might be processed (or transcriptionally terminated) at both its $5^{\prime}$ and $3^{\prime}$ ends.

\section{CJnc180, but not CJnc190, requires its antisense partner for processing by RNase III}

As the RNase III-dependent biosynthesis pathway of CJnc190 and CJnc180 make them distinct from other processed sRNAs characterized in Gammaproteobacteria, we next explored their maturation in more detail. Because of their extensive complementarity, we hypothesized that RNase III coprocesses CJnc180:CJnc190 duplexes. To examine this, we repeated northern blot analysis of the CJnc180/190 promoter inactivation allele strains, but this time in an RNase III+ background. This surprisingly showed that the C-190 only strain (with the CJnc180 promoter disrupted) still expressed mature CJnc190 (Fig 4A). We also found that CJnc190 precursors from either P1 or P2 could give rise to mature CJnc190, although when expressed from P1 or P2 alone, mature sRNA levels were approximately $60 \%$ of those detected in the WT, C-180/190, or C-190 only strains. Together, this suggests that both promoters drive transcription of pre-CJnc190 precursors and also that their combined activity in log phase contributes to levels of the mature sRNA.

We next examined whether CJnc180 processing by RNase III is likewise CJnc190independent. The $\Delta 180 / 190$ strain was complemented with pre-CJnc180 alone at $r d x A$ (strain C-180; both CJnc190 promoters disrupted). Surprisingly, and in contrast to CJnc190, CJnc180 was not processed without its antisense partner (Fig 4B, lane 4). Instead, as in $\Delta r n c$, we detected only pre-CJnc180. To confirm that CJnc180 processing requires pairing with CJnc190, we added back different CJnc190 species to the second unrelated Cj0046 pseudogene locus in the C-180 only strain (lanes 5-7). Expression of CJnc190 (either P1 or P2) from this second locus restored processing of CJnc180, and even in trans expression of 
“mature” CJnc190 from the C-190(Proc) strain (which overlaps the CJnc180 3' end) was sufficient to restore CJnc180 processing (lane 9). Overall, these mutational analyses indicate that while CJnc190 is processed independently of CJnc180, CJnc180 processing is mediated by expression of CJnc190 in cis or trans.

\section{CJnc190 processing is mediated by an intramolecular duplex}

To understand how CJnc190 might be processed without CJnc180, we performed folding predictions for six precursors supported by our 5' and 3' mapping (Fig S10). This indicated that the most abundant CJnc190 precursor (pre-CJnc190186, from P2) could fold into a long duplex flanking the mature sRNA, with an additional 5' hairpin (Fig 5A). Predicted structures of four additional precursors included the same $18 \mathrm{bpt}$ hairpin, but as part of more extended duplexes (Fig S10B). Primer extension analysis of CJnc190 in an RNase III+ background detected two CJnc190 5' ends, in addition to those corresponding to the P1/P2 transcriptional start sites, located in this hairpin that were RNase III dependent and CJnc180 independent (Fig S9B \& Fig 5A, sites a/b). These positions do not reflect the mature CJnc190 5' end and northern blot analysis in strains expressing CJnc190 from either of these sites (a or b) showed that CJnc190 is still processed in an RNase IIIdependent fashion (Fig S11A, lanes 3/4 \& 7/8), suggesting that the hairpin is not required for processing. We therefore next examined the requirement of the extended $5^{\prime}-3^{\prime}$ end duplex flanking the mature sRNA for processing.

To test if the $3^{\prime}$ end is required for processing by RNase III, we truncated the $3^{\prime}$ end of pre-CJnc190 to the position of the mature sRNA. Unlike a $5^{\prime}$ end truncation to the mature CJnc190 end, this abolished detection of mature CJnc190 (Fig 5B, lanes 3/4 \& 5/6). 
Truncation of either hairpin intermediate precursors at their $3^{\prime}$ end also abolished processing (Fig S11A, lanes 5/6 \& 9/10). In contrast, the mature sRNA was detected when the $3^{\prime}$ end was removed from the $5^{\prime}$-truncated version (Fig $\mathbf{5 B}$, lanes 7 \& 8), suggesting the unprocessed sRNA is not stable. Only CJnc190 precursors with a $3^{\prime}$ extension beyond the mature sRNA showed base-pairing between $5^{\prime}$ and $3^{\prime}$ precursor ends (Fig S10B). Together, this suggests that RNase III cleaves at both ends to generate mature CJnc190, and provides insight into how processing is independent of CJnc180. However, based on mature CJnc190 ends detected in WT, the processing steps following RNase III cleavage remain to be determined.

\section{CJnc190 drives processing of CJnc180}

We next examined why CJnc180 was not processed without CJnc190. Compared to preCJnc190, the predicted secondary structures of the major 154 nt (Fig 5C) or 104 nt preCJnc180 (Fig S12A) species do not contain distinct long duplexes like pre-CJnc190. We next performed in vitro RNase III cleavage assays with T7-transcribed pre-CJnc180154 in the absence or presence of mature CJnc190 to see if we could recapitulate processing in vitro. In contrast to in vivo, RNase III could cleave pre-CJnc180 even without CJnc190, although the cleavage site was located $\sim 100 \mathrm{nt}$ from the $5^{\prime}$ end (Fig. 5D, site d), rather than at the RNase III-dependent $3^{\prime}$ end of the mature sRNA detected in vivo (Fig 5C, site e). In contrast, reactions with increasing amounts of mature CJnc190 generated the in vivo RNase IIIdependent cleavage site (Fig 5D, site e), as well as a second site (Fig S12A, site f). Together, our data suggest that while RNase III is sufficient for maturation of structured CJnc190, CJnc180 also requires its antisense partner for processing. Finally, we searched for 
evidence of co-processing of the two sRNAs in vivo. However, inspection of CJnc190 primer extension analysis (Fig S9B) did not reveal any RNase III- and CJnc180-dependent 5' ends that would be consistent with the 2 nt 3'-overhangs generated by RNase III (Fig 5C, bottom, site c). Since this would disrupt SL1, which potentially protects the sRNA from RNase-mediated degradation, this suggests that the CJnc190 product of co-processing might be unstable. Alternatively, only the CJnc180 strand of the duplex might be cleaved, as has been reported for some RNase III substrates (Court et al, 2013) and appears to be the case for the pre-CJnc190 hairpin.

\section{CJnc180 indirectly affects ptmG via CJnc190 antagonism}

Since CJnc180 expression is conserved in C. jejuni and C. coli strains (Riedel et al, 2020; Dugar et al, 2013) but was not required for CJnc190 processing, its function remained unclear. Because of its extensive complementarity to CJnc190, as well as its co-processing, we hypothesized that it might serve as a CJnc190 antagonist and indirectly affect ptmG regulation. Examination of CJnc180, CJnc190, and ptmG in WT over growth showed that mature CJnc190 and ptmG levels were inversely correlated (Fig 6A). Moreover, mature CJnc190 accumulated while levels of pre-CJnc180 decreased as culture density increased. Processed CJnc180 levels also mildly decreased. Despite these inverse relationships, abolishing CJnc180 expression in log phase did not significantly affect PtmG-3×FLAG levels, although CJnc190 levels were very mildly increased (Fig 6B, lane 3 vs. lane 4; Fig S13A). However, in strains with only a single CJnc190 promoter intact [C-190(P1) or C-190(P2)], target levels were intermediate between WT and $\Delta 180 / 190$, in line with the $\sim 2$-fold difference in mature CJnc190 levels in these strains (Fig 6B, lanes 5 \& 6; Fig S13A). 
As 2-fold modulation of CJnc190 levels was sufficient to effect ptmG regulation in log phase, we next examined the effect of CJnc180 overexpression (second copy of full-length CJnc180 or "pre-processed" CJnc180-rrnB at $r d x A$; Fig 1D) on PtmG regulation. Western blot analysis showed that overexpression of CJnc180 or CJnc180(Proc) increased PtmG$3 \times$ FLAG to levels intermediate between $\Delta 180 / 190$ and WT (Fig 6C). In WT, the ratio of CJnc190 to all CJnc180 transcripts in log phase was 35:1 (Fig S13B). CJnc180 overexpression decreased this ratio to $\sim 13: 1$. This suggests that the asRAA can in fact influence $p t m G$, indirectly via an effect on CJnc190.

We next examined the effect of CJnc180 on CJnc190-mediated ptmG regulation at different phases of growth. PtmG-3×FLAG levels were compared at early exponential, mid exponential, and early stationary phases in $\Delta 180 / 190, \mathrm{C}-180 / 190, \mathrm{C}-190-$ only, and OE180(Proc) strains. PtmG-3×FLAG target levels were relatively stable in the C-180/190 complemented strain at all culture densities, and in $\Delta 180 / 190$ showed sustained upregulation. CJnc180 absence or overexpression affected PtmG::3×FLAG levels, depending on growth phase (Figs 6D \& S13C). For C-190-only (no CJnc180), levels were mildly decreased only in early stationary (lane 12). In contrast, overexpression of CJnc180 derepressed PtmG-3×FLAG levels in mid-log (lane 7) but had no significant effect in stationary phase. Together, these experiments provide evidence that levels of PtmG are affected by relatively mild changes in mature CJnc190 levels and/or availability, and also that CJnc180 is an example of a cis-acting sRNA antagonist. Importantly, they were performed without strong overexpression, and therefore might reflect physiologically relevant regulatory scenarios. 


\section{DISCUSSION}

Here, we have shown that the virulence-associated CJnc180/190 locus of the food-borne pathogen C. jejuni (Alzheimer et al, 2020) encodes a pair of sRNAs encoded antisense to each other with a complex biogenesis pathway involving RNase III. We demonstrated that one of these sRNAs, CJnc190, acts as a direct post-transcriptional repressor of ptmG, encoding a flagellin modification factor that is required for colonization (Alzheimer et al, 2020; Howard et al, 2009), and that this regulation is antagonized by the cis-encoded CJnc180 sRNA. Although the sRNAs are expressed antisense to each other and both are processed by RNase III, only processing of CJnc180 requires its antisense partner, and CJnc190 is processed independently of CJnc180. Overall, our study of CJnc190 provides insight into post-transcriptional regulation in $C$. jejuni, as well as more generally into the complex cross-talk among RNA molecules and the role of RNase III in sRNA biogenesis.

\section{Role of RNase III in sRNA biogenesis}

While many enterobacterial sRNAs are generated or activated via processing by RNase E (Chao et al, 2017), our study revealed RNase III as a crucial factor for CJnc190 sRNA biogenesis in $C$. jejuni. In $S$. aureus, RsaC sRNA is generated by RNase III from the 3' UTR of the mntABC mRNA (Lalaouna et al, 2019) by an unknown mechanism, although it was suggested that one of two detected asRNAs might drive its processing. Surprisingly, CJnc190 processing was independent of its antisense RNA CJnc180. Instead, CJnc190 maturation involves co-processing on both sides of the mature sRNA by RNase III, via a long duplex region involving both ends of the precursor in predicted secondary structures of pre-CJnc190. The processing of an sRNA by the dsRNA-specific RNase III from an 
extended duplex is reminiscent of miRNA processing by Dicer/Drosha in eukaryotes (Lee et al, 2003; Bernstein et al, 2001). Our data show that RNase III is clearly required for CJnc190 maturation, which in turn is required for $p t m G$ regulation. However, it remains unclear why CJnc190 has this complex biogenesis pathway. Processing seems to generate a more stable form of CJnc190, but might also affect its activity. For mRNAs, RNase III processing of stem-loop structures in $5^{\prime}$ UTRs has been reported to impact their translation and/or mediate autocontrol of rnc itself (Court et al, 2013). As it remains unclear how cleavage of the long CJnc190 5'/3' end duplex might give rise to the final mature sRNA, additional enzymes such as RNase J, Y, R, or PNPase might be involved in further processing or trimming to the mature $5^{\prime} / 3^{\prime}$ ends. For example, in E. coli, processing of ribosomal RNAs or prophage-encoded DicF is initiated by RNase III cleavage of a stem-loop, followed by additional cleavages by RNases such as RNase E (Faubladier \& Bouché, 1994; Shajani et al, 2011), and RNase III cleavage is followed by RNase J1 trimming during processing of the SRP RNA component scRNA (small cytoplasmic RNA) in B. subtilis (Yao et al, 2007). Additional pre-CJnc190 mutant analysis together with in vitro RNase III cleavage assays, or combinations of RNase mutations in vivo, will allow a more complete description of CJnc190 biogenesis and show if players other than RNase III, including trans-encoded RNAs, are also involved.

In contrast to CJnc190, CJnc180 processing by RNase III required its cis-encoded partner to create a double-stranded substrate. The toxin mRNAs of several type I toxinantitoxin systems have long been known to be processed by RNase III upon interaction with their antitoxin RNAs (Vogel et al, 2004; Gerdes et al, 1992) in some bacteria such as Bacillus even underlying its essentiality (Durand et al. 2012). Besides processing of type I TA 
loci, RNase III has been implicated in degradation and processing of diverse sense/antisense RNA pairs, including those encoded on plasmids (Blomberg et al, 1990), antisense RNA mediated mRNA processing (Opdyke et al, 2011), ribosomal RNA processing (Iost et al, 2019), sRNA-mRNA target pair co-degradation (Viegas et al, 2011; Romilly et al, 2012; Afonyushkin et al, 2005), and CRISPR/tracrRNA co-processing by RNase III in Cas9based CRISPR/Cas systems (Deltcheva et al, 2011; Dugar et al, 2013).

Considering sRNA biogenesis, RNase E has the most well-characterized role in bacteria (Miyakoshi et al, 2015a; Bandyra \& Luisi, 2018) but is absent in 1/5 sequenced strains (Hui et al, 2014). Despite its broader conservation, understanding of the role of RNase III in sRNA biogenesis lags behind that of RNase E. However, genome-wide surveys

in diverse bacteria have identified many potential sRNAs as RNase III targets that remain to be studied (DiChiara et al, 2016; Stead et al, 2011; Altuvia et al, 2018; Lioliou et al, 2012, 2013; Le Rhun et al, 2017; Gatewood et al, 2012; Lybecker et al, 2014; Vogel et al, 2003), suggesting that the role of RNase III in sRNA biogenesis might be larger than previously appreciated.

\section{CJnc190 is antagonized by the cis-encoded CJnc180 sRNA}

While CJnc180 is dispensable for CJnc190 processing and $p t m G$ repression, we have provided in vivo evidence that CJnc180 is a cis-acting antagonist of CJnc190 that can affect ptmG regulation. CJnc180 therefore appears to be a new cis-acting representative of the socalled "sponge" RNAs that target sRNAs (reviewed in (Denham, 2020; Figueroa-Bossi \& Bossi, 2018; Grüll \& Massé, 2019)). Such sponges have mainly been reported to be transencoded and can be derived from mRNAs, tRNA 3' external transcribed spacers or other 
sRNAs. In contrast, CJnc180 is antagonizing a cis-encoded sRNA and additional candidates for sponge RNAs are cis-encoded pairs expressed under different conditions (Denham, 2020). For example, antisense SraC/SdsR (RyeA/RyeB), widely conserved in E. coli and Salmonella (Fröhlich et al, 2016), show reciprocal expression and are also processed by RNase III (Vogel et al, 2003). In E. coli, SdsR overexpression leads to cell death via repression of $y h c B$, which is rescued by SraC overexpression, and the pair were also proposed as a novel toxin-antitoxin system where both components are sRNAs (Choi et al, 2018; Gupta et al, 2019; Choi et al, 2019). Whether the cis-acting antagonist CJnc180 from the CJnc190/180 locus also has an additional function and can act on other RNAs in trans remains to be seen.

The potential for independent regulation of CJnc180 and CJnc190 makes an antagonistic relationship attractive. While the exact mechanism of antagonism is unclear, hypotheses can be made based on other cis-acting sRNAs (Brantl, 2007). CJnc180:CJnc190 co-processing in SL1 suggests antagonism could occur via cleavage and decay. Since overexpression of either "pre-processed" or full-length CJnc180 affected ptmG, coprocessing might not be the only mechanism by which CJnc180 influences CJnc190. The extensive complementarity remaining for the processed sRNAs means that even mature CJnc180 could sequester CJnc190, followed by degradation by RNase III, or transcriptional interference might be involved. The mechanism by which CJnc180 affects CJnc190 might also be dependent on the stoichiometry of the two sRNAs (Denham, 2020), for example, as the sRNAs change in relative levels over growth similar as has been reported for the ChiX sRNA and its sponge, the $c h b B C$ intergenic region (Figueroa-Bossi et al, 2009). Presumably, CJnc180 could serve to buffer and/or set a threshold for CJnc190 levels to regulate targets, 
as for tRNA-derived sponge RNAs in E. coli (Lalaouna et al, 2015). While we observed that the mature sRNAs show inverse expression levels during growth, it remains unknown how they are transcriptionally controlled and in response to which signals their levels change. While CJnc180 is transcribed from one promoter (P1), CJnc190 is transcribed from at least two promoters (P1\&P2). Precursors from both promoters are expressed during routine growth and give rise to the mature sRNA. The two promoters presumably increase the potential for environmental inputs into CJnc180/190, thereby increasing the complexity of the locus even further. Future work will reveal transcriptional regulators and conditions that regulate the CJnc180/190 promoters, and how transcriptional control intersects with RNase III processing.

\section{CJnc190 directly represses ptmG by targeting a G-rich sequence and impacts virulence}

Based on in vitro and in vivo analyses, we demonstrated that CJnc190 directly represses ptmG translation by base-pairing with the G-rich RBS of its mRNA using a C/U-rich loop. Thus, CJnc190 binding likely interferes with translation initiation, resembling the canonical mode of sRNA-mediated target repression (Storz et al, 2011). The structure of mature CJnc190 is reminiscent of RepG sRNA from H. pylori, which uses a C/U-rich loop to target a phase-variable G-repeat in the 5'UTR of a chemotaxis receptor mRNA (Pernitzsch et al, 2014), indicating they might be functional homologs. Also in other species several sRNAs with C/U-rich target interaction sites have been reported (Bronesky et al, 2016; Heidrich et al, 2017; Schmidtke et al, 2013; Boisset et al, 2007), suggesting that targeting of G-rich sequences might be a more widespread phenomenon. Although CJnc190 and RepG share 
$\mathrm{C} / \mathrm{U}$-rich loop regions, their biogenesis is strikingly different: while RepG is transcribed as a separate standing gene corresponding to the mature sRNA (Pernitzsch et al, 2014), CJnc190 is transcribed opposite to another sRNA (CJnc180) and is processed into the mature form from diverse precursors. It is interesting to imagine how CJnc190 (and CJnc180) might have arisen - de novo (Jose et al, 2019), or from a degenerate type I toxinantitoxin system (Balbontín et al, 2008). For example, a sRNA from a predicted type I toxinantitoxin system acts as a trans-acting sRNA repressor of the major virulence genes of $H$. pylori (Eisenbart et al, 2020).

Our previous study of CJnc180/190 in our 3D model of the human intestine (Alzheimer et al, 2020), showed that CJnc180/190 as well as ptmG, the target of CJnc190, are involved in infection. PtmG is part of a six-gene cluster in the flagellin glycosylation island of $C$. jejuni NCTC11168 that has been associated with livestock strains (Champion et al., 2005; Mourkas et al., 2020) and is in the pathway generating legionaminic acid sugar precursors that decorate its flagellins (Howard et al, 2009; Zebian et al, 2016). As PtmG is required for chicken colonization (Howard et al, 2009), CJnc190 might impact virulence phenotypes via $p t m G$ regulation. However, as CJnc190 is also found in strains that lack PtmG such as strain 81-176 (Dugar et al, 2013), it likely has additional targets that remain to be identified that could account for its infection phenotype (Alzheimer et al., 2020). In addition, CJnc180, for which we showed an antagonizing function on CJnc190-mediated repression of $p t m G$, might have an additional function as an sRNA targeting trans-encoded mRNAs and thereby impact virulence. Future studies will reveal the complete regulon of each sRNA and the contribution of their direct targets to virulence phenotypes. 


\section{MATERIALS AND METHODS}

Bacterial strains and culture conditions. C. jejuni strains (Table S2) were routinely grown either on Müller-Hinton (MH) agar plates or with shaking at $140 \mathrm{rpm}$ in Brucella broth $(\mathrm{BB})$ at $37^{\circ} \mathrm{C}$ in a microaerobic atmosphere $\left(10 \% \mathrm{CO}_{2}, 5 \% \mathrm{O}_{2}\right)$. All C. jejuni media was supplemented with $10 \mu \mathrm{g} / \mathrm{ml}$ vancomycin. Agar was also supplemented with markerselective antibiotics [20 $\mu \mathrm{g} / \mathrm{ml}$ chloramphenicol, $50 \mu \mathrm{g} / \mathrm{ml}$ kanamycin (Kan), $20 \mu \mathrm{g} / \mathrm{ml}$ gentamicin (Gm), or $250 \mu \mathrm{g} / \mathrm{ml}$ hygromycin B (Hyg)] where appropriate. E. coli strains were grown aerobically at $37^{\circ} \mathrm{C}$ in Luria-Bertani (LB) broth or on LB agar supplemented with the appropriate antibiotics for marker selection.

General recombinant DNA techniques. Oligonucleotide primers for PCR, site-directed mutagenesis, Sanger sequencing, and northern blot probing are listed in Table $\mathbf{S} \mathbf{3}$ and were purchased from Sigma. Plasmids generated and/or used in this study are listed in Table S4. Site-directed mutagenesis was performed on plasmids by inverse PCR with mutagenic primers as listed in Table S5, according to standard protocols. DNA constructs and mutations were confirmed by Sanger sequencing (Macrogen). Restriction enzymes, Taq polymerase for validation PCR, and T4 DNA ligase were purchased from NEB. For cloning purposes, Phusion high-fidelity DNA polymerase (Thermo Fisher Scientific) was used. For PCR amplification of constructs containing the HygR cassette, 3\% DMSO was included in reactions.

Total RNA extraction and analysis by northern blotting. For analysis of total RNA, bacterial strains were grown to log phase in BB and approximately $4 \mathrm{OD}_{600}$ were harvested and mixed with 0.2 volumes of stop-mix (95\% ethanol and 5\% phenol, v/v). Samples were 
immediately snap-frozen in liquid nitrogen and stored at $-80^{\circ} \mathrm{C}$ until RNA extraction.

Frozen samples were thawed on ice and centrifuged at $4^{\circ} \mathrm{C}$ to collect cell pellets $(4,500 \mathrm{~g}$, $20 \mathrm{~min}$ ), which were then lysed by resuspension in $600 \mu \mathrm{l}$ of a solution containing $0.5 \mathrm{mg} / \mathrm{ml}$ lysozyme and 1\% SDS in Tris-EDTA buffer (pH 8.0) and incubation for $2 \mathrm{~min}$ at $64^{\circ} \mathrm{C}$. Total RNA was extracted from the lysate using the hot-phenol method as described previously (Sharma et al, 2010). For northern blot analysis, 5 - $10 \mu \mathrm{g}$ of total RNA in Gel Loading Buffer II (GLII, Ambion) was loaded per lane on 6\% polyacrylamide (PAA) / 7 M urea denaturing gels in $1 \times$ TBE buffer. Following electrophoretic separation, RNA was transferred to Hybond-XL membranes (GE Healthcare) by electroblotting. Transferred RNA was then cross-linked to the membrane with ultraviolet light to the membrane and hybridized with $\gamma^{32}$ P-ATP end-labelled DNA oligonucleotides (Table S3) in Roti Hybriquick (Roth) at $42^{\circ} \mathrm{C}$ overnight. Membranes were then washed 20 minutes each at $42^{\circ} \mathrm{C}$ in $5 \times, 1 \times$, and $0.5 \times$ SSC (saline-sodium citrate) $+0.1 \%$ SDS, dried, and exposed to a phosphorimager screen. Screens were scanned using a FLA-3000 Series Phosphorimager (Fuji) and bands were quantified using AIDA software (Raytest, Germany).

Total protein sample analysis by SDS-PAGE and western blotting. Analysis of protein expression in $C$. jejuni was performed by SDS-PAGE and western blotting. Bacterial cells were collected from cultures in mid-log phase (OD 600 0.4-0.5) by centrifugation at $11,000 \mathrm{~g}$ for $3 \mathrm{~min}$. Cell pellets were resuspended in $100 \mu \mathrm{l}$ of $1 \times$ protein loading buffer $(62.5 \mathrm{mM}$ Tris-HCl, pH 6.8, 100 mM DTT, 10\% (v/v) glycerol, 2\% (w/v) SDS, 0.01\% (w/v) bromophenol blue) and boiled for $8 \mathrm{~min}$. For analysis of total proteins, $0.05-0.1$ OD 600 of cells were loaded per lane on a 12\% SDS-polyacrylamide gels. Gels were stained with 
PageBlue (Thermo Fisher Scientific, \#24620). For western blot analysis, samples corresponding to an OD600 of 0.05-0.1 were separated on 12\% SDS-PAA gels and transferred to a nitrocellulose membrane by semidry blotting. Membranes were blocked for $1 \mathrm{~h}$ with $10 \%$ (w/v) milk powder in TBS-T (Tris-buffered saline-Tween-20) and then incubated overnight with primary antibody (monoclonal anti-FLAG, 1:1,000; SigmaAldrich, \#F1804-1MG; or anti-GFP, 1:1000, Roche \#11814460001) at $4^{\circ} \mathrm{C}$. Membranes were then washed with TBS-T, followed by $1 \mathrm{~h}$ incubation with secondary antibody (antimouse IgG, 1:10,000 in 3\% BSA/TBS-T; GE-Healthcare, \#RPN4201). All antibodies were dissolved in 3\% BSA (bovine serum albumin)/TBS-T. After washing, the blot was developed using enhanced chemiluminescence reagent and an ImageQuant LAS-4000 (GE). Bands were quantified using AIDA software. As a loading control, a monoclonal antibody specific for GroEL (1:10,000; Sigma-Aldrich, \# G6532-5ML) with an anti-rabbit IgG (1:10,000; GE Healthcare, \#RPN4301) secondary antibody was used to probe membranes after FLAG/GFP.

Primer extension analysis of RNA $5^{\prime}$ ends. Total RNA was extracted from bacteria in log phase as described above. RNA was digested with DNase I (Thermo Fisher Scientific) to remove DNA, and then 5 - $10 \mu \mathrm{g}$ of RNA was added to a total volume of $5.5 \mu \mathrm{l}$ with $\mathrm{H}_{2} \mathrm{O}$, denatured, and snap-cooled on ice. A 5' end ${ }^{32}$ P labeled DNA oligonucleotide complementary to the RNA of interest was then added (Table S3) and annealed by heating to $80^{\circ} \mathrm{C}$, followed by slow cooling $\left(1^{\circ} \mathrm{C}\right.$ per min) to $42^{\circ} \mathrm{C}$. A master mix with reverse transcriptase (RT) buffer and 20 U Maxima RT (Thermo Fisher Scientific) was added and the reaction was allowed to proceed for $1 \mathrm{~h}$ at $50^{\circ} \mathrm{C}$. Reactions were stopped with $12 \mu \mathrm{l}$ GLII 
(Ambion, 95\% (v/v) formamide, 18 mM EDTA, and 0.025\% (w/v) SDS, xylene cyanol, and bromophenol blue). A sequencing ladder was also constructed using the DNA Cycle sequencing kit (Jena Bioscience) according to the manufacturer's instructions with the CJnc180/190 region amplified with primers CSO-0354/0355 (NCTC11168 wild-type) from genomic DNA as template and the same radioactively-labeled primer used for the reverse transcription reaction. Reactions separated on 6 or $10 \%$ PAA-urea sequencing gels, which were then dried and exposed to a phosphorimager screen, and then scanned (FLA-3000 Series, Fuji). The following primers were used for primer extension: CJnc190- CSO-0185; CJnc180 - CSO-0188.

In vitro transcription and $5^{\prime}$ end-labeling of RNAs. PCR with Phusion polymerase was used to generate DNA templates containing the T7 promoter sequence using oligonucleotides and DNA templates listed in Table S7. Transcription of RNAs in vitro by T7 RNA polymerase was then carried out using the MEGAscript T7 kit (Ambion) according to the manufacturer's instructions. RNAs were then checked for quality by electrophoresis on a PAA-urea gel, dephosphorylated with Antarctic Phosphatase (NEB), 5' -end-labelled $\left(\gamma^{32} \mathrm{P}\right)$ with polynucleotide kinase (ThermoFisher Scientific), and purified by gel extraction as previously described (Papenfort et al, 2006). Expected sequences of the resulting T7 transcripts are listed in Table S7.

Electrophoretic mobility shift assays (EMSAs). Gel-shift assays were performed as described previously (Pernitzsch et al, 2014). Briefly, 5' end radiolabeled RNA (0.04 pmol) was denatured $\left(1 \mathrm{~min}, 95^{\circ} \mathrm{C}\right)$ and cooled for $5 \mathrm{~min}$ on ice. Yeast tRNA ( $1 \mu \mathrm{g}$, Ambion) and $1 \mu \mathrm{l}$ of 10× RNA Structure Buffer (Final concentration $10 \mathrm{mM}$ Tris, pH 7, $100 \mathrm{mM} \mathrm{KCl,}$ 
$10 \mathrm{mM} \mathrm{MgCl}$ ) was then mixed with the labelled RNA. Unlabeled RNA ( $2 \mu$ l diluted in 1× Structure Buffer) was added to the desired final concentrations ( $0 \mathrm{nM}, 10 \mathrm{nM}, 20 \mathrm{nM}$, $50 \mathrm{nM}, 100 \mathrm{nM}, 200 \mathrm{nM}, 500 \mathrm{nM}$, or $1 \mu \mathrm{M}$ ). Binding reactions were incubated at $37^{\circ} \mathrm{C}$ for 15 min. Before loading on a pre-cooled native 6\% PAA, $0.5 \times$ TBE gel, samples were mixed with $3 \mu$ l native loading buffer [50\% (v/v) glycerol, $0.5 \times$ TBE, $0.2 \%(\mathrm{w} / \mathrm{v})$ bromophenol blue]. Gels were run in $0.5 \times \mathrm{TBE}$ buffer at $300 \mathrm{~V}$ and $4^{\circ} \mathrm{C}$. Gels were dried, exposed to a phosphorimager screen, and then scanned (FLA-3000 Series, Fuji).

Inline probing. Inline probing assays for RNA structure and binding interactions in vitro were performed essentially as described previously (Pernitzsch et al, 2014). End-labeled RNAs ( 0.2 pmol, see above) in $5 \mu$ l water were mixed with an equal volume of $2 \times$ Inline buffer (100 mM Tris-HCl, pH 8.3, $40 \mathrm{mM} \mathrm{MgCl}_{2}$, and $200 \mathrm{mM} \mathrm{KCl}$ and incubated for $40 \mathrm{~h}$ at room temperature to allow spontaneous cleavage. Reactions were stopped with an equal volume of $2 \times$ Colourless loading buffer (10 M urea and 1.5 mM EDTA, pH 8.0). Reactions were separated on 6 or 10\% PAA-urea sequencing gels, which were dried and exposed to a PhosphorImager screen. RNA ladders were prepared using Alkaline hydrolysis buffer $(\mathrm{OH}$ ladder) or Sequencing buffer (T1 ladder) according to the manufacturer's instructions (Ambion).

RNase III cleavage assays. In vitro-transcribed pre-CJnc180 was 5' end labeled as described for Inline probing and EMSA and subjected to RNase III cleavage assays as follows. Labeled pre-CJnc180 (0.2 pmol) was briefly denatured and snap cooled on ice, followed by the addition of Structure buffer to a final concentration of $1 \times$ and yeast tRNA to a final concentration of $0.1 \mathrm{mg} / \mathrm{ml}$. Where necessary, unlabeled mature CJnc190 0.2 or 2 
pmol) was denatured and snap-cooled separately and added to reactions. Reactions were pre-incubated at $37^{\circ} \mathrm{C}$ for 10 minutes, followed by the addition of RNase III (NEB; $1 / 625 \mathrm{U}$ ) and further incubation at $37^{\circ} \mathrm{C}$ for 5 minutes to allow limited cleavage. Reactions were stopped by the addition of an equal volume of GLII and separated on a 10\% PAA-urea sequencing gel, which was then dried and exposed to a PhosphorImager screen. RNA ladders were prepared using Alkaline hydrolysis buffer (OH ladder) or Sequencing buffer (T1 ladder) according to the manufacturer's instructions (Ambion).

In vitro translation. In vitro translation of target $m R N A$ reporter fusions in the presence and absence of sRNAs was carried out using the PURExpress system (NEB). An in vitrotranscribed RNA including the $p t m G 5^{\prime}$ leader (including RBS and CJnc190 binding site) and first 10 codons fused to gfpmut3 was used as template for translation (Table S7). For each reaction, 4 pmol of denatured template RNA was incubated in either alone or with equimolar $(1 \times), 10 \times$, or $50 \times$ ratios of sRNA species for 10 minutes at $37^{\circ} \mathrm{C}$. In vitro translation components were then added, and reactions were incubated a further 2 hours at $37^{\circ} \mathrm{C}$. Reactions were stopped with an equal volume of $2 \times$ protein loading buffer. One half of the reaction was analyzed by western blotting on $12 \%$ SDS-PAA gels with an antibody against GFP, and the second half was loaded on a second gel which was stained with PageBlue after electrophoresis, as a loading control.

Differential RNA-seq data. Processed primary transcriptome data generated by dRNA-seq for C. jejuni NCTC11168 (Dugar et al, 2013) was retrieved from the NCBI Gene Expression Omnibus (GEO) using the accession GSE38883, was inspected using the Integrated Genome Browser (bioviz.org) (Freese et al, 2016). 


\section{ACKNOWLEDGEMENTS}

We are grateful to Monika Raabe for acquiring mass spectrometry data. We thank Sharma lab members for fruitful discussions on this project and Lydia Hadjeras, Mona Alzheimer, and Elisabetta Fiore for critical comments on the manuscript.

\section{CONFLICT OF INTEREST}

The authors declare that they have no conflict of interest.

\section{AUTHOR CONTRIBUTIONS}

SLS \& CMS designed the experiments; SLS performed the experiments; SLS \& CMS analyzed data; SLS \& CMS wrote the manuscript. 


\section{REFERENCES}

Adams PP, Baniulyte G, Esnault C, Chegireddy K, Singh N, Monge M, Dale RK, Storz G \& Wade JT (2021) Regulatory roles of Escherichia coli 5' UTR and ORF-internal RNAs detected by 3' end mapping. elife 10:

Adams PP \& Storz G (2020) Prevalence of small base-pairing RNAs derived from diverse genomic loci. Biochim. Biophys. Acta Gene Regul. Mech. 1863: 194524

Afonyushkin T, Vecerek B, Moll I, Bläsi U \& Kaberdin VR (2005) Both RNase E and RNase III control the stability of $\operatorname{sod} B$ mRNA upon translational inhibition by the small regulatory RNA RyhB. Nucleic Acids Res. 33: 1678-1689

Altuvia Y, Bar A, Reiss N, Karavani E, Argaman L \& Margalit H (2018) In vivo cleavage rules and target repertoire of RNase III in Escherichia coli. Nucleic Acids Res. 46: 10530-10531

Alzheimer M, Svensson SL, König F, Schweinlin M, Metzger M, Walles H \& Sharma CM (2020) A three-dimensional intestinal tissue model reveals factors and small regulatory RNAs important for colonization with Campylobacter jejuni. PLoS Pathog. 16: e1008304

Bandyra KJ \& Luisi BF (2018) RNase E and the High-Fidelity Orchestration of RNA Metabolism. Microbiol. Spectr. 6(2): doi: 10.1128/microbiolspec.RWR-0008-2017.

Bernstein E, Caudy AA, Hammond SM \& Hannon GJ (2001) Role for a bidentate ribonuclease in the initiation step of RNA interference. Nature 409: 363-366

Blomberg P, Wagner EG \& Nordström K (1990) Control of replication of plasmid R1: the duplex between the antisense RNA, CopA, and its target, CopT, is processed specifically in vivo and in vitro by RNase III. EMBO J. 9: 2331-2340

Boisset S, Geissmann T, Huntzinger E, Fechter P, Bendridi N, Possedko M, Chevalier C, Helfer AC, Benito Y, Jacquier A, Gaspin C, Vandenesch F \& Romby P (2007) Staphylococcus aureus RNAIII coordinately represses the synthesis of virulence factors and the transcription regulator Rot by an antisense mechanism. Genes Dev. 21: 1353-1366

Brantl S (2007) Regulatory mechanisms employed by cis-encoded antisense RNAs. Curr. Opin. Microbiol. 10: 102-109

Bronesky D, Desgranges E, Corvaglia A, François P, Caballero CJ, Prado L, Toledo-Arana A, Lasa I, Moreau K, Vandenesch F, Marzi S, Romby P \& Caldelari I (2019) A multifaceted small RNA modulates gene expression upon glucose limitation in Staphylococcus aureus. EMBO J. 38:

Bronesky D, Wu Z, Marzi S, Walter P, Geissmann T, Moreau K, Vandenesch F, Caldelari I \& Romby P (2016) Staphylococcus aureus RNAIII and Its Regulon Link Quorum Sensing, Stress Responses, Metabolic Adaptation, and Regulation of Virulence Gene Expression. Annu. Rev. Microbiol. 70: 299-316

Burnham PM \& Hendrixson DR (2018) Campylobacter jejuni: collective components promoting a successful enteric lifestyle. Nat. Rev. Microbiol. 16: 551-565

Carthew RW \& Sontheimer EJ (2009) Origins and Mechanisms of miRNAs and siRNAs. Cell 136: 
642-655

Chae H, Han K, Kim K, Park H, Lee J \& Lee Y (2011) Rho-dependent termination of ssrS (6S RNA) transcription in Escherichia coli: implication for 3' processing of 6S RNA and expression of downstream ygfA (putative 5-formyl-tetrahydrofolate cyclo-ligase). J. Biol. Chem. 286: 114122

Chao Y, Li L, Girodat D, Förstner KU, Said N, Corcoran C, Śmiga M, Papenfort K, Reinhardt R, Wieden H-J, Luisi BF \& Vogel J (2017) In Vivo Cleavage Map Illuminates the Central Role of RNase E in Coding and Non-coding RNA Pathways. Mol. Cell 65: 39-51

Chao Y, Papenfort K, Reinhardt R, Sharma CM \& Vogel J (2012) An atlas of Hfq-bound transcripts reveals 3' UTRs as a genomic reservoir of regulatory small RNAs. EMBO J. 31: 4005-4019

Chao Y \& Vogel J (2016) A 3' UTR-Derived Small RNA Provides the Regulatory Noncoding Arm of the Inner Membrane Stress Response. Mol. Cell 61: 352-363

Choi JS, Kim W, Suk S, Park H, Bak G, Yoon J \& Lee Y (2018) The small RNA, SdsR, acts as a novel type of toxin in Escherichia coli. RNA Biol.

Choi JS, Park H, Kim W \& Lee Y (2019) Coordinate regulation of the expression of SdsR toxin and its downstream pphA gene by RyeA antitoxin in Escherichia coli. Sci. Rep. 9: 9627

Court DL, Gan J, Liang Y-H, Shaw GX, Tropea JE, Costantino N, Waugh DS \& Ji X (2013) RNase III: genetics and function; structure and mechanism. Annu. Rev. Genet. 47: 405-431

Davis BM \& Waldor MK (2007) RNase E-dependent processing stabilizes MicX, a Vibrio cholerae sRNA. Mol. Microbiol. 65: 373-385

Deltcheva E, Chylinski K, Sharma CM, Gonzales K, Chao Y, Pirzada ZA, Eckert MR, Vogel J \& Charpentier E (2011) CRISPR RNA maturation by trans-encoded small RNA and host factor RNase III. Nature 471: 602-607

Denham EL (2020) The Sponge RNAs of bacteria - How to find them and their role in regulating the post-transcriptional network. Biochim. Biophys. Acta Gene Regul. Mech. 1863: 194565

De Mets F, Van Melderen L \& Gottesman S (2019) Regulation of acetate metabolism and coordination with the TCA cycle via a processed small RNA. Proc Natl Acad Sci USA 116: 10431052

DiChiara JM, Liu B, Figaro S, Condon C \& Bechhofer DH (2016) Mapping of internal monophosphate 5 ' ends of Bacillus subtilis messenger RNAs and ribosomal RNAs in wild-type and ribonucleasemutant strains. Nucleic Acids Res. 44: 3373-3389

Dugar G, Herbig A, Förstner KU, Heidrich N, Reinhardt R, Nieselt K \& Sharma CM (2013) Highresolution transcriptome maps reveal strain-specific regulatory features of multiple Campylobacter jejuni isolates. PLoS Genet. 9: e1003495

Dugar G, Leenay RT, Eisenbart SK, Bischler T, Aul BU, Beisel CL \& Sharma CM (2018) CRISPR RNADependent Binding and Cleavage of Endogenous RNAs by the Campylobacter jejuni Cas9. Mol. Cell 69: 893-905.e7

Durand S, Gilet L \& Condon C (2012) The essential function of B. subtilis RNase III is to silence 
foreign toxin genes. PLoS Genet. 8: e1003181

Eisenbart SK, Alzheimer M, Pernitzsch SR, Dietrich S, Stahl S \& Sharma CM (2020) A RepeatAssociated Small RNA Controls the Major Virulence Factors of Helicobacter pylori. Mol. Cell 80: 210-226.e7

Faubladier M \& Bouché JP (1994) Division inhibition gene dicF of Escherichia coli reveals a widespread group of prophage sequences in bacterial genomes. J. Bacteriol. 176: 1150-1156

Figueroa-Bossi N \& Bossi L (2018) Sponges and predators in the small RNA world. Microbiol. Spectr. 6(4). doi: 10.1128/microbiolspec.RWR-0021-2018.

Figueroa-Bossi N, Valentini M, Malleret L, Fiorini F \& Bossi L (2009) Caught at its own game: regulatory small RNA inactivated by an inducible transcript mimicking its target. Genes Dev. 23: $2004-2015$

Freese NH, Norris DC \& Loraine AE (2016) Integrated genome browser: visual analytics platform for genomics. Bioinformatics 32: 2089-2095

Fröhlich KS, Haneke K, Papenfort K \& Vogel J (2016) The target spectrum of SdsR small RNA in Salmonella. Nucleic Acids Res. 44: 10406-10422

Gatewood ML, Bralley P, Weil MR \& Jones GH (2012) RNA-Seq and RNA immunoprecipitation analyses of the transcriptome of Streptomyces coelicolor identify substrates for RNase III. J. Bacteriol. 194: 2228-2237

Gerdes K, Nielsen A, Thorsted P \& Wagner EG (1992) Mechanism of killer gene activation. Antisense RNA-dependent RNase III cleavage ensures rapid turn-over of the stable hok, srnB and pndA effector messenger RNAs. J. Mol. Biol. 226: 637-649

Gordon GC, Cameron JC \& Pfleger BF (2017) RNA Sequencing Identifies New RNase III Cleavage Sites in Escherichia coli and Reveals Increased Regulation of mRNA. MBio 8:

Grüll MP \& Massé E (2019) Mimicry, deception and competition: The life of competing endogenous RNAs. Wiley Interdiscip. Rev. RNA 10: e1525

Guo MS, Updegrove TB, Gogol EB, Shabalina SA, Gross CA \& Storz G (2014) MicL, a new $\sigma^{\mathrm{E}}$ dependent sRNA, combats envelope stress by repressing synthesis of Lpp, the major outer membrane lipoprotein. Genes Dev. 28: 1620-1634

Gupta AK, Siddiqui N, Yadav D, Arora L \& Dutta T (2019) Regulation of RyeA/SraC expression in Escherichia coli. Biochem. Biophys. Res. Commun. 516: 661-665

Haddad N, Saramago M, Matos RG, Prévost H \& Arraiano CM (2013) Characterization of the biochemical properties of Campylobacter jejuni RNase III. Biosci. Rep. 33:

Havelaar AH, Kirk MD, Torgerson PR, Gibb HJ, Hald T, Lake RJ, Praet N, Bellinger DC, de Silva NR, Gargouri N, Speybroeck N, Cawthorne A, Mathers C, Stein C, Angulo FJ, Devleesschauwer B \& World Health Organization Foodborne Disease Burden Epidemiology Reference Group (2015) World health organization global estimates and regional comparisons of the burden of foodborne disease in 2010. PLoS Med. 12: e1001923

Heidrich N, Bauriedl S, Barquist L, Li L, Schoen C \& Vogel J (2017) The primary transcriptome of 
Neisseria meningitidis and its interaction with the RNA chaperone Hfq. Nucleic Acids Res. 45: 6147-6167

Holmqvist E \& Vogel J (2018) RNA-binding proteins in bacteria. Nat. Rev. Microbiol. 16: 601-615

Hör J, Garriss G, Di Giorgio S, Hack L-M, Vanselow JT, Förstner KU, Schlosser A, Henriques-Normark B \& Vogel J (2020) Grad-seq in a Gram-positive bacterium reveals exonucleolytic sRNA activation in competence control. EMBO J. 39: e103852

Howard SL, Jagannathan A, Soo EC, Hui JPM, Aubry AJ, Ahmed I, Karlyshev A, Kelly JF, Jones MA, Stevens MP, Logan SM \& Wren BW (2009) Campylobacter jejuni glycosylation island important in cell charge, legionaminic acid biosynthesis, and colonization of chickens. Infect. Immun. 77: 2544-2556

Hui MP, Foley PL \& Belasco JG (2014) Messenger RNA degradation in bacterial cells. Annu. Rev. Genet. 48: 537-559

Iost I, Chabas S \& Darfeuille F (2019) Maturation of atypical ribosomal RNA precursors in Helicobacter pylori. Nucleic Acids Res.

Kavita K, de Mets F \& Gottesman S (2018) New aspects of RNA-based regulation by Hfq and its partner sRNAs. Curr. Opin. Microbiol. 42: 53-61

Lalaouna D, Baude J, Wu Z, Tomasini A, Chicher J, Marzi S, Vandenesch F, Romby P, Caldelari I \& Moreau K (2019) RsaC sRNA modulates the oxidative stress response of Staphylococcus aureus during manganese starvation. Nucleic Acids Res. 47: 9871-9887

Lalaouna D, Carrier M-C, Semsey S, Brouard J-S, Wang J, Wade JT \& Massé E (2015) A 3' external transcribed spacer in a tRNA transcript acts as a sponge for small RNAs to prevent transcriptional noise. Mol. Cell 58: 393-405

Le Rhun A, Lécrivain A-L, Reimegård J, Proux-Wéra E, Broglia L, Della Beffa C \& Charpentier E (2017) Identification of endoribonuclease specific cleavage positions reveals novel targets of RNase III in Streptococcus pyogenes. Nucleic Acids Res. 45: 2329-2340

Lee Y, Ahn C, Han J, Choi H, Kim J, Yim J, Lee J, Provost P, Rådmark O, Kim S \& Kim VN (2003) The nuclear RNase III Drosha initiates microRNA processing. Nature 425: 415-419

Lioliou E, Sharma CM, Altuvia Y, Caldelari I, Romilly C, Helfer A-C, Margalit H \& Romby P (2013) In vivo mapping of RNA-RNA interactions in Staphylococcus aureus using the endoribonuclease III. Methods 63: 135-143

Lioliou E, Sharma CM, Caldelari I, Helfer A-C, Fechter P, Vandenesch F, Vogel J \& Romby P (2012) Global regulatory functions of the Staphylococcus aureus endoribonuclease III in gene expression. PLoS Genet. e1002782

Lorenz R, Bernhart SH, Höner Zu Siederdissen C, Tafer H, Flamm C, Stadler PF \& Hofacker IL (2011) ViennaRNA Package 2.0. Algorithms Mol. Biol. 6: 26

Lybecker M, Zimmermann B, Bilusic I, Tukhtubaeva N \& Schroeder R (2014) The double-stranded transcriptome of Escherichia coli. Proc Natl Acad Sci USA 111: 3134-3139

Mann M, Wright PR \& Backofen R (2017) IntaRNA 2.0: enhanced and customizable prediction of 
RNA-RNA interactions. Nucleic Acids Res. 45: W435-W439

Melamed S, Adams PP, Zhang A, Zhang H \& Storz G (2020) RNA-RNA Interactomes of ProQ and Hfq Reveal Overlapping and Competing Roles. Mol. Cell 77: 411-425.e7

Miyakoshi M, Chao Y \& Vogel J (2015a) Regulatory small RNAs from the 3' regions of bacterial mRNAs. Curr. Opin. Microbiol. 24: 132-139

Miyakoshi M, Chao Y \& Vogel J (2015b) Cross talk between ABC transporter mRNAs via a target mRNA-derived sponge of the GcvB small RNA. EMBO J. 34: 1478-1492

Opdyke JA, Fozo EM, Hemm MR \& Storz G (2011) RNase III participates in GadY-dependent cleavage of the gadX-gadW mRNA. J. Mol. Biol. 406: 29-43

Papenfort K, Espinosa E, Casadesús J \& Vogel J (2015) Small RNA-based feedforward loop with AND-gate logic regulates extrachromosomal DNA transfer in Salmonella. Proc Natl Acad Sci USA 112: E4772-81

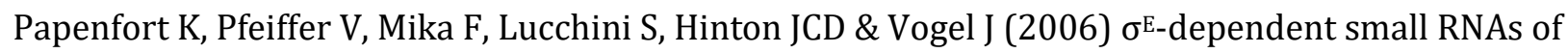
Salmonella respond to membrane stress by accelerating global omp mRNA decay. Mol. Microbiol. 62: 1674-1688

Papenfort K, Said N, Welsink T, Lucchini S, Hinton JCD \& Vogel J (2009) Specific and pleiotropic patterns of mRNA regulation by ArcZ, a conserved, Hfq-dependent small RNA. Mol. Microbiol. 74: $139-158$

Parkhill J, Wren BW, Mungall K, Ketley JM, Churcher C, Basham D, Chillingworth T, Davies RM, Feltwell T, Holroyd S, Jagels K, Karlyshev AV, Moule S, Pallen MJ, Penn CW, Quail MA, Rajandream MA, Rutherford KM, van Vliet AH, Whitehead S, et al (2000) The genome sequence of the food-borne pathogen Campylobacter jejuni reveals hypervariable sequences. Nature 403: $665-668$

Pernitzsch SR \& Sharma CM (2012) Transcriptome complexity and riboregulation in the human pathogen Helicobacter pylori. Front. Cell. Infect. Microbiol. 2: 14

Pernitzsch SR, Tirier SM, Beier D \& Sharma CM (2014) A variable homopolymeric G-repeat defines small RNA-mediated posttranscriptional regulation of a chemotaxis receptor in Helicobacter pylori. Proc Natl Acad Sci USA 111: E501-10

Quendera AP, Seixas AF, Dos Santos RF, Santos I, Silva JPN, Arraiano CM \& Andrade JM (2020) RNABinding Proteins Driving the Regulatory Activity of Small Non-coding RNAs in Bacteria. Front. Mol. Biosci. 7: 78

Quereda JJ \& Cossart P (2017) Regulating Bacterial Virulence with RNA. Annu. Rev. Microbiol. 71: 263-280

Rath EC, Pitman S, Cho KH \& Bai Y (2017) Identification of streptococcal small RNAs that are putative targets of RNase III through bioinformatics analysis of RNA sequencing data. $B M C$ Bioinformatics 18: 540

Riedel C, Förstner KU, Püning C, Alter T, Sharma CM \& Gölz G (2020) Differences in the Transcriptomic Response of Campylobacter coli and Campylobacter lari to Heat Stress. Front. 
Microbiol. 11: 523

Romeo T \& Babitzke P (2018) Global regulation by CsrA and its RNA antagonists. Microbiol. Spectr. 6(2): doi:10.1128/microbiolspec.RWR-0009-2017

Romilly C, Chevalier C, Marzi S, Masquida B, Geissmann T, Vandenesch F, Westhof E \& Romby P (2012) Loop-loop interactions involved in antisense regulation are processed by the endoribonuclease III in Staphylococcus aureus. RNA Biol. 9: 1461-1472

Schmidtke C, Abendroth U, Brock J, Serrania J, Becker A \& Bonas U (2013) Small RNA sX13: a multifaceted regulator of virulence in the plant pathogen Xanthomonas. PLoS Pathog. 9: e1003626

Shajani Z, Sykes MT \& Williamson JR (2011) Assembly of bacterial ribosomes. Annu. Rev. Biochem. 80: $501-526$

Sharma CM, Hoffmann S, Darfeuille F, Reignier J, Findeiss S, Sittka A, Chabas S, Reiche K, Hackermüller J, Reinhardt R, Stadler PF \& Vogel J (2010) The primary transcriptome of the major human pathogen Helicobacter pylori. Nature 464: 250-255

Stead MB, Marshburn S, Mohanty BK, Mitra J, Pena Castillo L, Ray D, van Bakel H, Hughes TR \& Kushner SR (2011) Analysis of Escherichia coli RNase E and RNase III activity in vivo using tiling microarrays. Nucleic Acids Res. 39: 3188-3203

Storz G, Vogel J \& Wassarman KM (2011) Regulation by small RNAs in bacteria: expanding frontiers. Mol. Cell 43: 880-891

Svensson SL \& Sharma CM (2016) Small RNAs in bacterial virulence and communication. Microbiol. Spectr. 4(3): doi: 10.1128/microbiolspec.VMBF-0028-2015.

Thomason MK \& Storz G (2010) Bacterial antisense RNAs: how many are there, and what are they doing? Annu. Rev. Genet. 44: 167-188

Tomb JF, White O, Kerlavage AR, Clayton RA, Sutton GG, Fleischmann RD, Ketchum KA, Klenk HP, Gill S, Dougherty BA, Nelson K, Quackenbush J, Zhou L, Kirkness EF, Peterson S, Loftus B, Richardson D, Dodson R, Khalak HG, Glodek A, et al (1997) The complete genome sequence of the gastric pathogen Helicobacter pylori. Nature 388: 539-547

Tree JJ, Granneman S, McAteer SP, Tollervey D \& Gally DL (2014) Identification of bacteriophageencoded anti-sRNAs in pathogenic Escherichia coli. Mol. Cell 55: 199-213

Viegas SC, Silva IJ, Saramago M, Domingues S \& Arraiano CM (2011) Regulation of the small regulatory RNA MicA by ribonuclease III: a target-dependent pathway. Nucleic Acids Res. 39: 2918-2930

Vogel J, Argaman L, Wagner EGH \& Altuvia S (2004) The small RNA IstR inhibits synthesis of an SOS-induced toxic peptide. Curr. Biol. 14: 2271-2276

Vogel J, Bartels V, Tang TH, Churakov G, Slagter-Jäger JG, Hüttenhofer A \& Wagner EGH (2003) RNomics in Escherichia coli detects new sRNA species and indicates parallel transcriptional output in bacteria. Nucleic Acids Res. 31: 6435-6443

Wassarman KM (2018) 6S RNA, a global regulator of transcription. Microbiol. Spectr. 6(3): 
doi:10.1128/microbiolspec.RWR-0019-2018

Westermann AJ (2018) Regulatory RNAs in Virulence and Host-Microbe Interactions. Microbiol. Spectr. 6:

Yao S, Blaustein JB \& Bechhofer DH (2007) Processing of Bacillus subtilis small cytoplasmic RNA: evidence for an additional endonuclease cleavage site. Nucleic Acids Res. 35: 4464-4473

Young KT, Davis LM \& Dirita VJ (2007) Campylobacter jejuni: molecular biology and pathogenesis. Nat. Rev. Microbiol. 5: 665-679

Zebian N, Merkx-Jacques A, Pittock PP, Houle S, Dozois CM, Lajoie GA \& Creuzenet C (2016) Comprehensive analysis of flagellin glycosylation in Campylobacter jejuni NCTC 11168 reveals incorporation of legionaminic acid and its importance for host colonization. Glycobiology 26: 386-397 


\section{FIGURES}

A

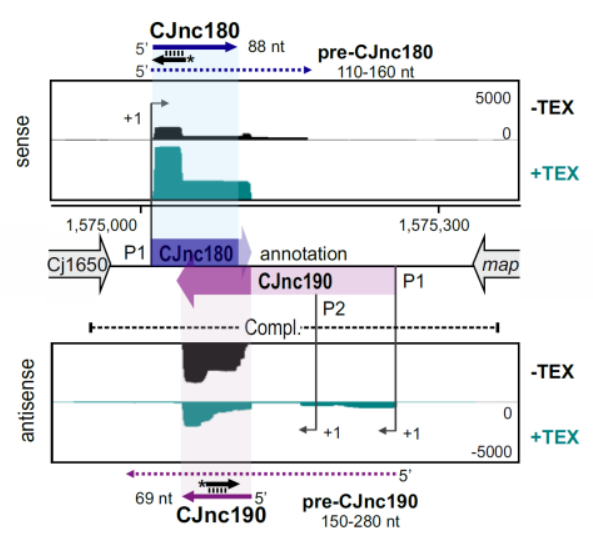

C

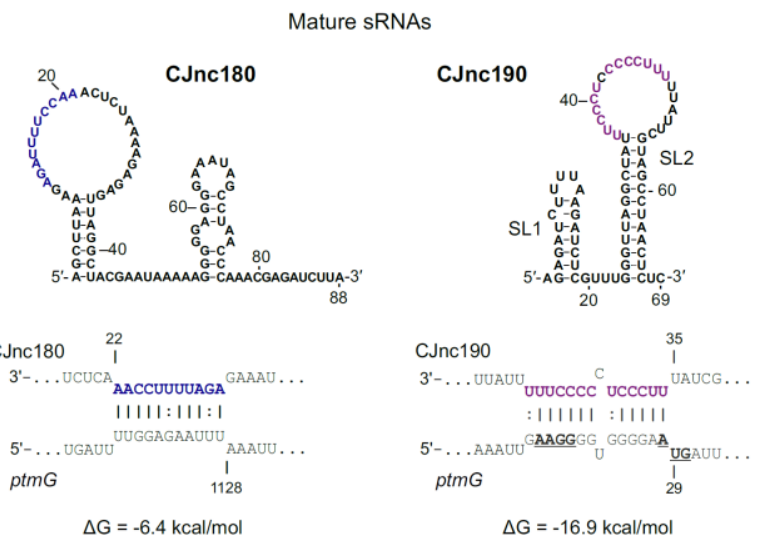

B

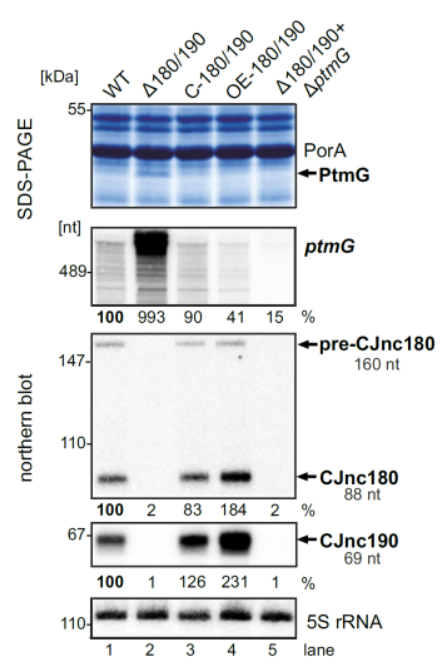

D

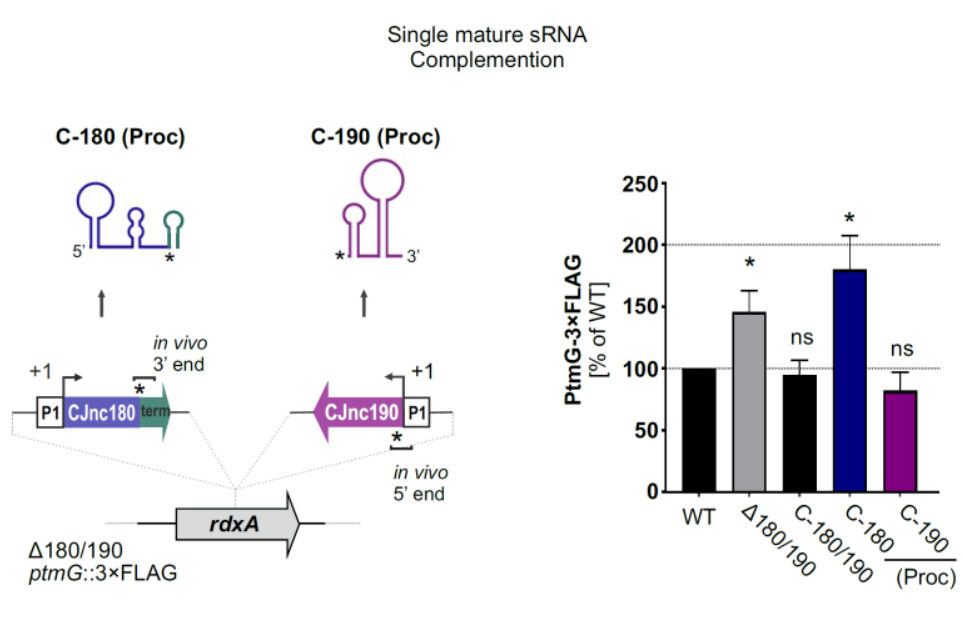

Figure 1. Processed CJnc190 represses ptmG expression. (A) dRNA-seq coverage (Dugar et al, 2013) for CJnc180 and CJnc190 sRNAs (annotated as 99 and 216 nt, respectively, coloured arrows) in $C$. jejuni NCTC11168. -/+TEX indicates dRNA-seq libraries generated from RNA -/+ terminator exonuclease treatment. Features identified in this work are labeled: +1 - transcription start sites; dashed/solid arrows (above/below) - precursor/mature sRNAs. Black dashed line: genomic region used for complementation (C-180/190). (B) Northern blot and SDS-PAGE analyses of RNA and protein from CJnc180/190 WT and mutant strains. Upregulated $\sim 45 \mathrm{kDa}$ PtmG and non-regulated PorA control are indicated. Probes for the mature sRNAs (starred arrows, panel A) and the 5' end of the ptmG ORF were used. OE, second-copy overexpression. (C) Predicted secondary structures (RNAfold) (Lorenz et al, 2011) and ptmG interactions (IntaRNA) (Mann et al, 2017) of mature CJnc180 and CJnc190. Blue/purple: potential ptmG pairing residues. Underlined: $p t m G$ RBS/start codon. (D) Complementation of $p t m G$ regulation in $\Delta 180 / 190$ with single mature sRNAs. (Left) To express CJnc180 only (C-180(Proc)), its mature 3' end was fused to the E. coli rrnB terminator; transcription is driven from its native promoter. For C-190(Proc), its mature $5^{\prime}$ end was fused to its annotated TSS (P1 promoter). (Right) PtmG-3×FLAG levels measured by western blotting. *: p<0.05, ns: not significant, vs. WT. See also Fig S3A. 
A

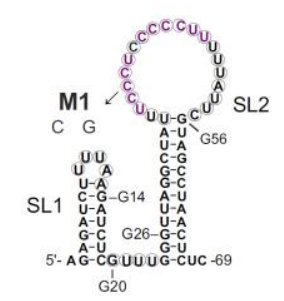

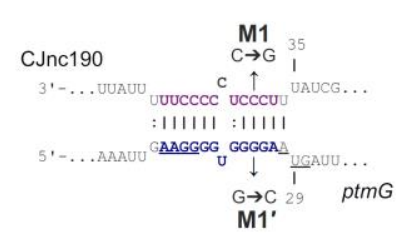

C

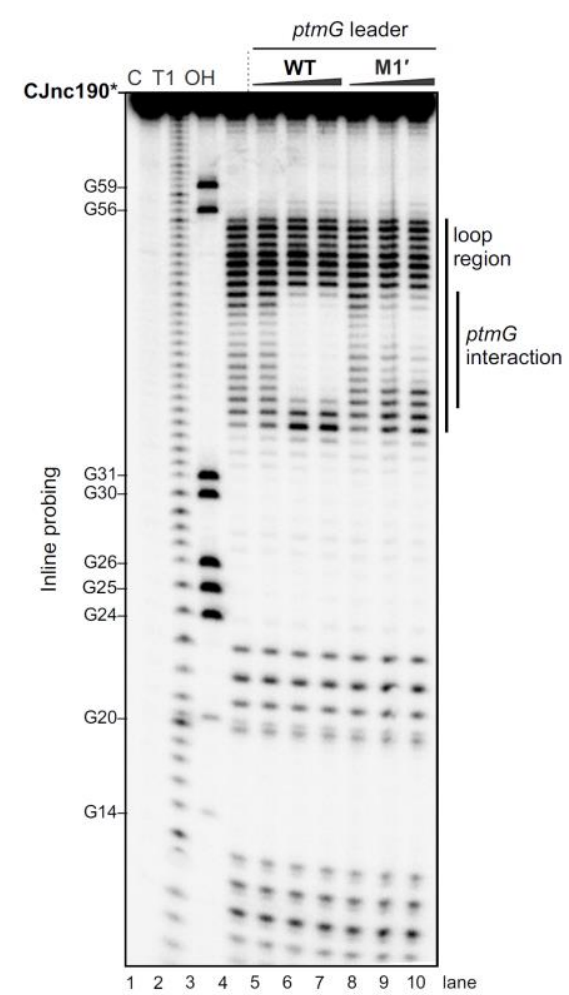

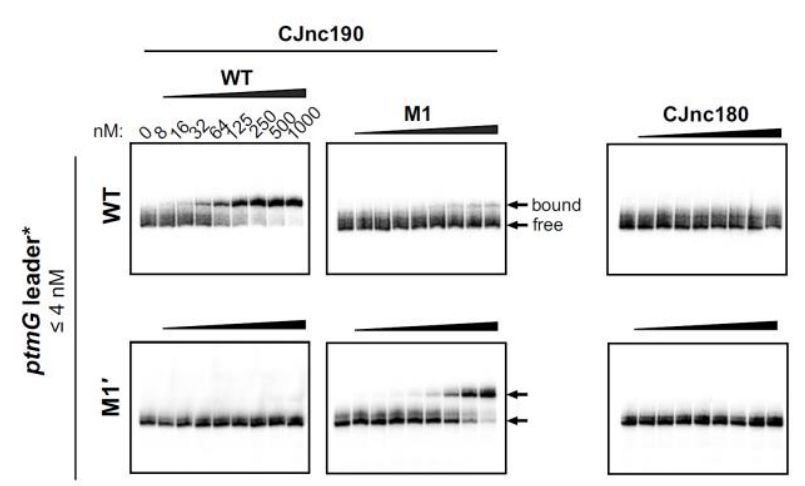

D

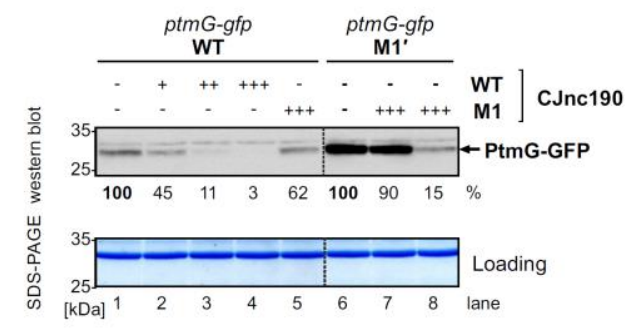

E

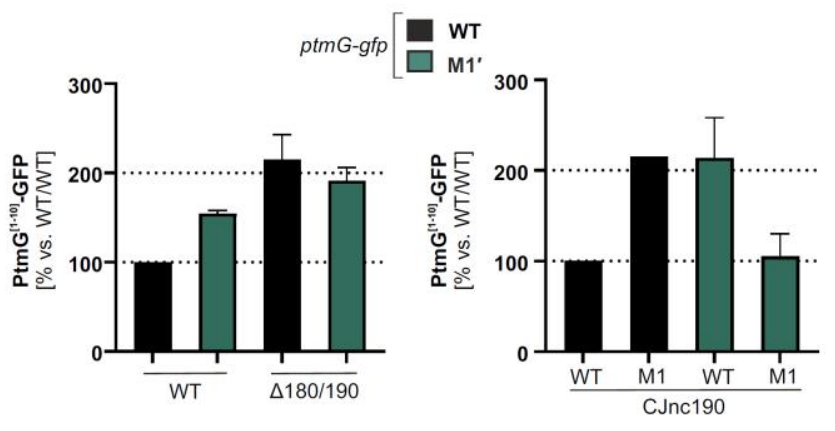

Figure 2. CJnc190 represses translation of ptmG via base-pairing with its G-rich RBS. (A) CJnc190 secondary structure based on inline probing (C) and interaction with the ptmG leader showing mutations (M1/M1') introduced into the interaction site. Circled residues: single-stranded regions mapped by inline probing. Blue/purple residues: $p t m G / C J n c 190$-protected nucleotides. RBS/start codon are underlined. (B) In vitro gel shift assay of ${ }^{32} \mathrm{P}-5^{\prime}$-labeled (marked with *) ptmG leader (WT/M1') with unlabeled CJnc190 WT/M1 as well as CJnc180 sRNAs. (C) Inline probing of 0.2 pmol ${ }^{32} \mathrm{P}-5$ '-end-labeled CJnc190 sRNA in the absence or presence of $0.2 / 2 / 20$ pmol cold ptmG leader (WT/M1'). C - untreated control; T1 ladder - G residues (indicated on left); $\mathrm{OH}$ - all positions (alkaline hydrolysis). (D) In vitro translation of a $p t m G$-GFP reporter (5' UTR and first 10 codons of $p t m G$ fused to gfpmut3, 2 pmol) in an E. coli cell-free system +/- CJnc190 (WT/M1 +: 2 pmol, ++: 20 pmol, +++: $100 \mathrm{pmol}$ ) detected by western blotting with an anti-GFP antibody. A stained gel of the same samples served as a loading control. (E) PtmG-GFP (WT/M1') reporter expression in vivo, +/- mature CJnc190 (WT/M1). PtmG-GFP levels are the mean of three independent replicates, with error bars representing the SEM. See also Fig S4C. 
A

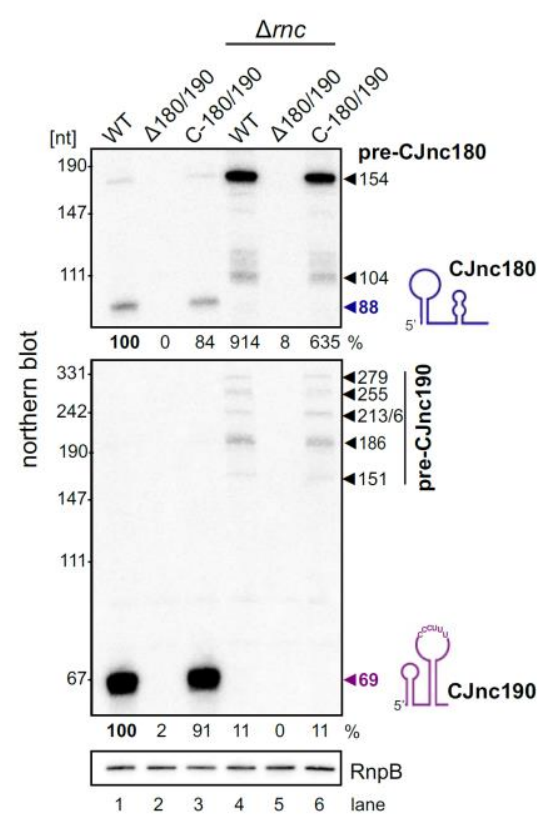

B

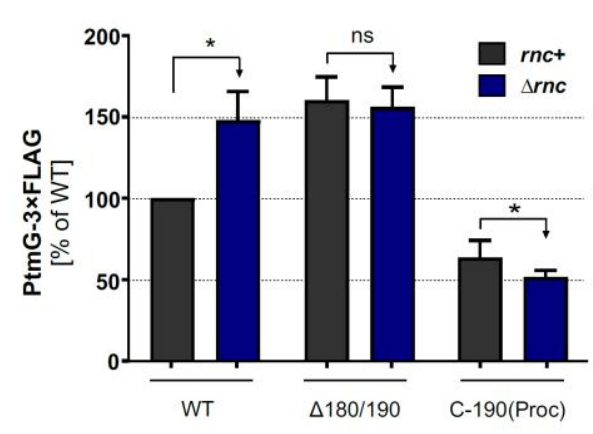

C
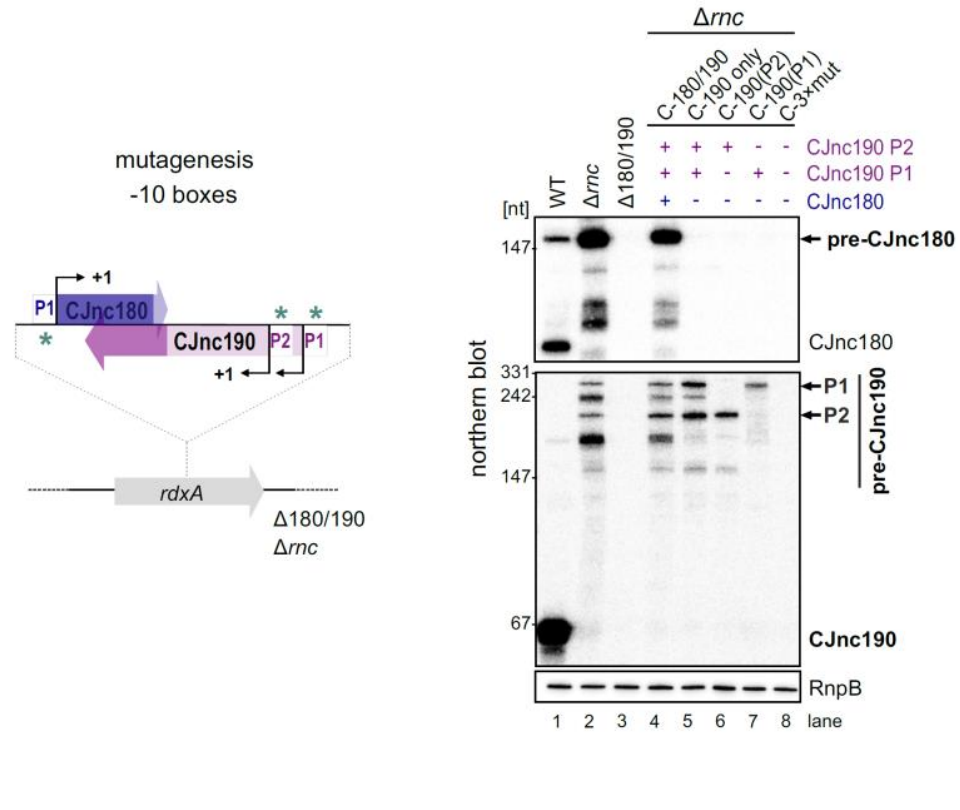

D

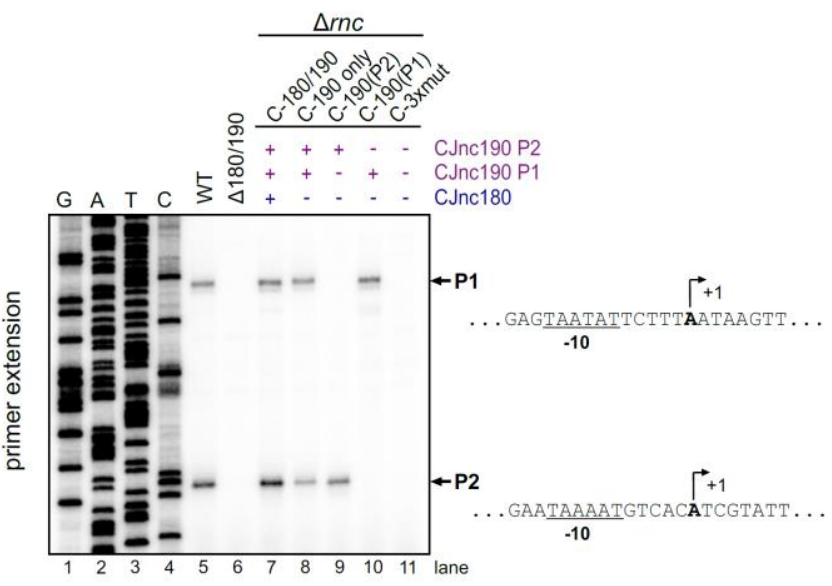

Figure 3. RNase III processes CJnc190 precursors expressed from two promoters. (A) Northern blot of CJnc180 and CJnc190 processing by RNase III in total RNA. Lengths are based on primer extension and 3' RACE. Quantification is for all bands combined. (B) Effect of rnc (RNase III) deletion on PtmG-3×FLAG levels in the absence or presence of CJnc180/190 sRNAs. *: p<0.1, ns: not significant, Student's unpaired t-test $(\mathrm{n}=3)$. See also Fig S5B. (C) Validation of CJnc180/190 promoters. (Left) Strategy for testing CJnc180/190 promoter activity by complementation of $\Delta 180 / 190$ with -10 box mutant alleles at $r d x A$. (Right) Northern blot analysis of preCJnc180/CJnc190 in sRNA promoter mutant strains ( $\Delta r n c$ background). (-/+): promoter mutant/WT. Probes for the mature sRNAs were used (CSO-0189/0185, respectively, for CJnc180/190; Fig 1A). RnpB served as loading control for northern blots. (D) Primer extension analysis of pre-CJnc190 5' ends in promoter mutant strains ( $\Delta r n c$ background). Total RNA was annealed with the probe for mature CJnc190 used for northern blots. A sequencing ladder was generated with the same probe (lanes 1-4). P1/P2: putative CJnc190 primary transcripts/5' ends. The full gel is shown in Fig S8A. 
A

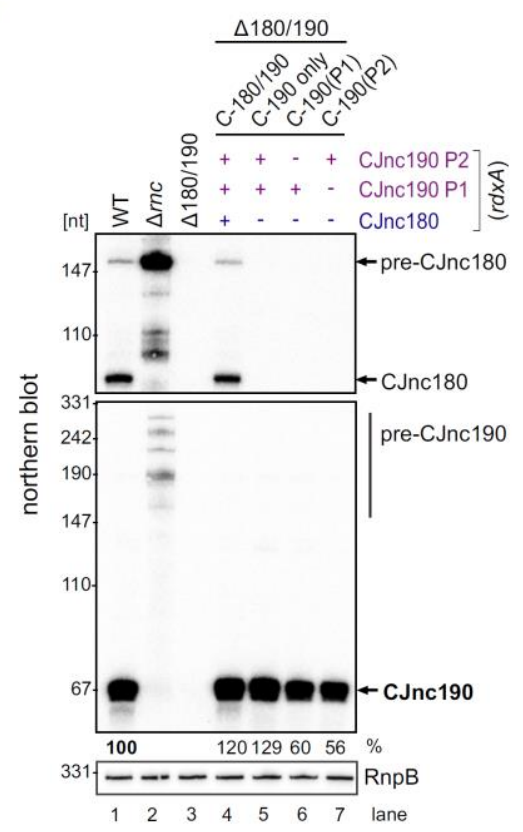

B

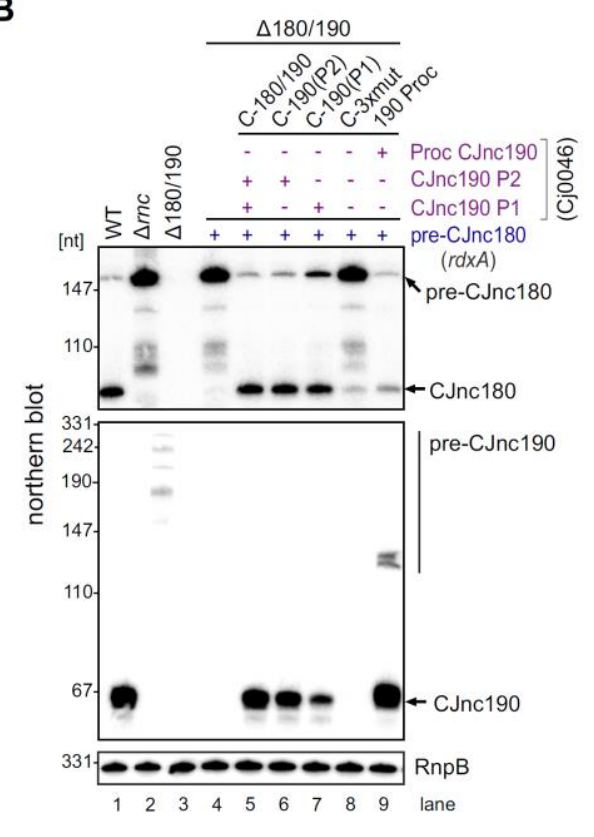

Figure 4. CJnc180 requires its antisense partner for RNase III-mediated processing, while CJnc190 processing is CJnc180-independent. (A) Northern blot analysis of pre-CJnc190 processing in vivo $+/$ - CJnc180. The $\Delta 180 / 190$ strain was complemented at $r d x A$ with WT or CJnc180/190 promoter mutant alleles in an $r n c+$ background. (B) Pre-CJnc180 processing in the presence or absence of CJnc190. Pre-CJnc180 was introduced into $r d x A$ of a $\Delta 180 / 190$ strain. Different CJnc190 species were expressed from the unrelated Cj0046 pseudogene locus. (+/-) indicates if a promoter in the CJnc180/190 allele is WT/mutant. For northern blot detection of CJnc180 and CJnc190, probes for the mature sRNAs were used (CSO-0189 and CSO-0185, respectively). RnpB (probed with CSO-0497) served as a loading control. 


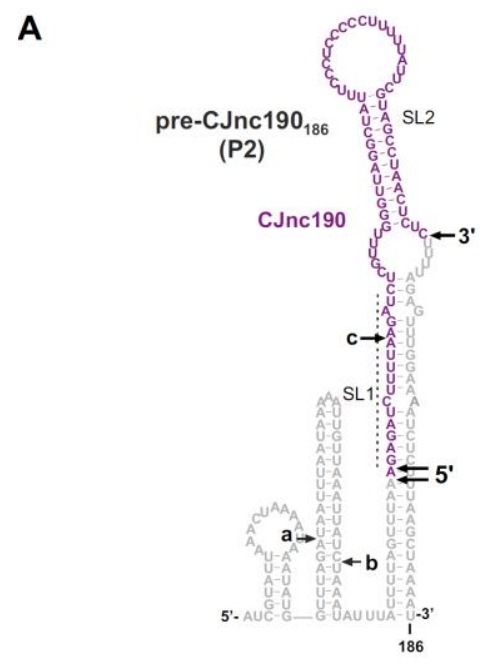

C
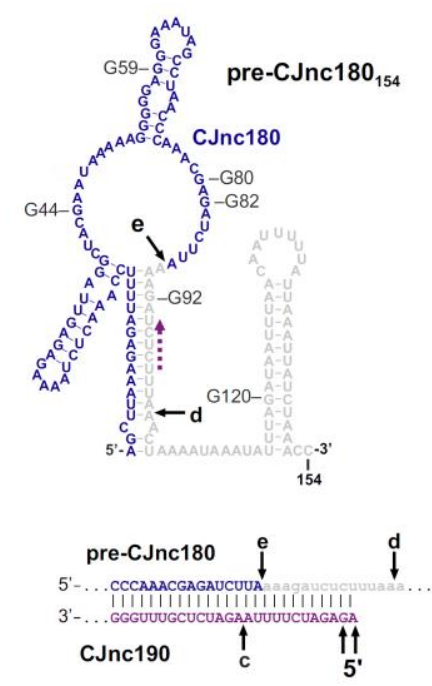

B

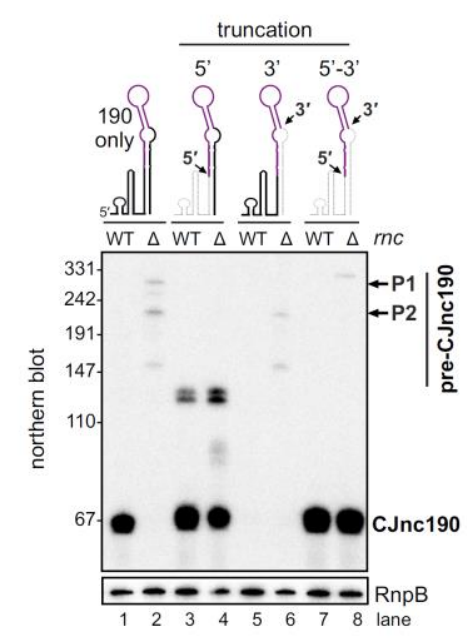

D

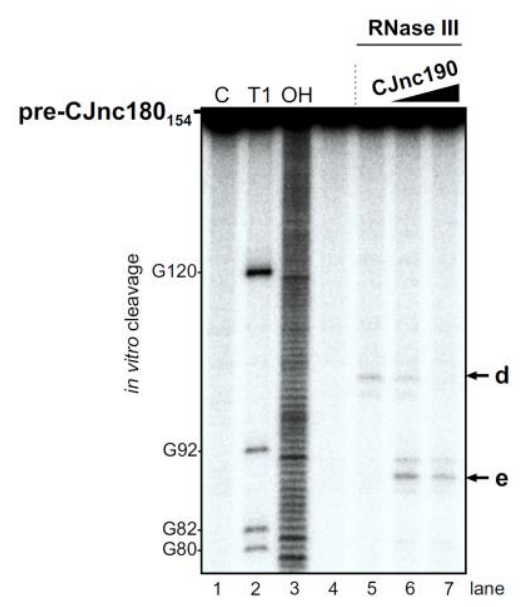

Figure 5. Intra- or intermolecular duplex mediated processing of the CJnc190 and CJnc180 SRNAs. (A) Predicted secondary structure of the pre-CJnc190 ${ }_{186}$ precursor transcribed from P2. 5' $/ 3^{\prime}$ indicates mature sRNA ends; A/B: putative intermediate $5^{\prime}$ ends identified by primer extension. C: putative co-processing site with CJnc180 (see panel C, bottom). (B) Both 5' and 3' ends of CJnc190 are required for processing in vivo. CJnc190 was expressed from the $r d x A$ locus in the $\Delta 180 / 190$ background as the WT version (CJnc190 only) or three versions with truncations at the mature sRNA 5' or 3' ends: I, 5' end truncation; II: 3' end truncation; III: both ends truncated. CJnc190 expression and processing was measured by northern blotting. A probe for mature CJnc190 was used (CSO0185), while RnpB (CSO-0497) served as a loading control. (C) (Top) Predicted secondary structure of pre-CJnc180 ${ }_{154}$. Site e: CJnc190-dependent CJnc180 3' end. Site d: CJnc190-independent in vitro cleavage site. Purple dashed arrow: $5^{\prime}$ end of mature CJnc190. (Bottom) Details of potential coprocessing of CJnc190 and pre-CJnc180. (D) In vitro RNase III cleavage of ${ }^{32} \mathrm{P} 5^{\prime}$ end labeled preCJnc180 ${ }_{154 .}{ }^{32} \mathrm{P}-5^{\prime}$-end labeled in vitro transcript ( $\left.0.2 \mathrm{pmol}\right)$ was incubated in the presence or absence of unlabeled mature CJnc190 ( 0.2 or $2 \mathrm{pmol})$ and subjected to cleavage with RNase III. C - untreated control; T1 ladder - G residues (indicated on left); $\mathrm{OH}$ - all positions (alkaline hydrolysis). The full gel is shown in Fig S12B. 
A

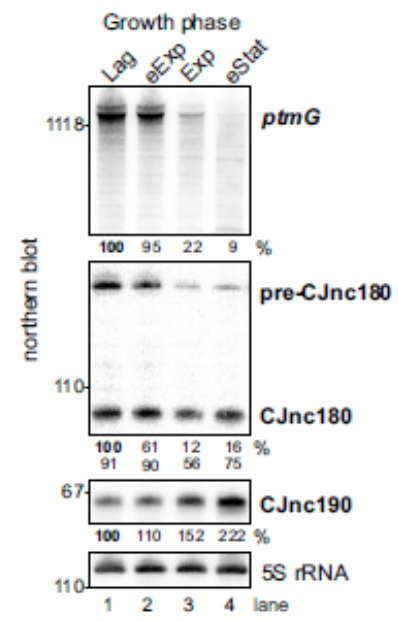

B

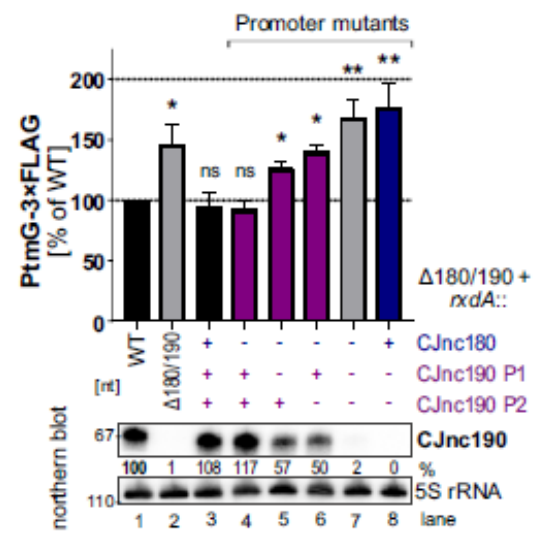

C
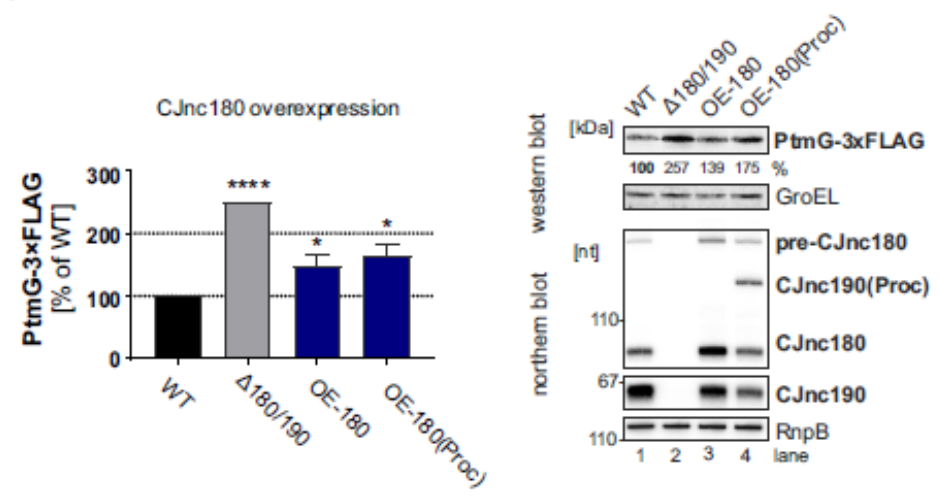

D

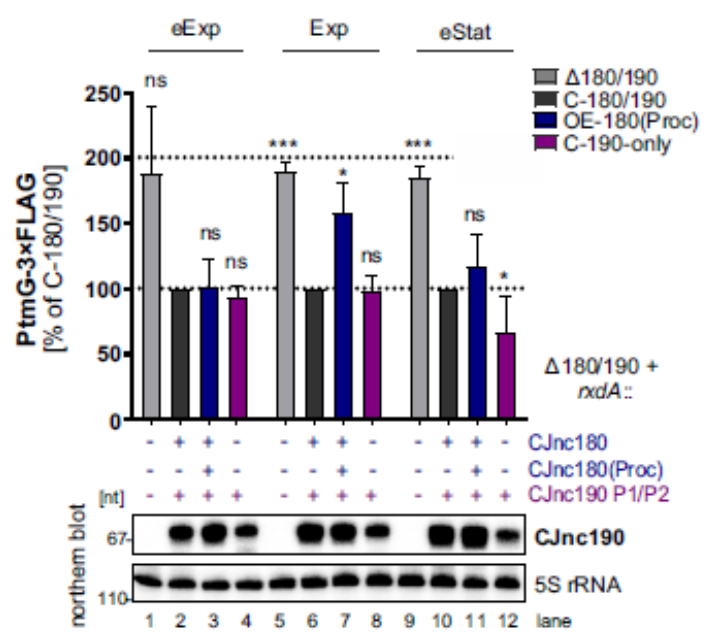

Figure 6. CJnc180 antagonizes CJnc190-mediated repression of ptmG. (A) Precursor, mature sRNA, and $p t m G$ target mRNA expression in WT at different growth phases in rich medium under microaerobic conditions. Lag: lag phase, eExp: early exponential, Exp: exponential, eStat: early stationary phase $\left(\mathrm{OD}_{600} 0.1,0.25,0.5\right.$, and 0.9 , respectively). (B) Expression of PtmG::3 $\times \mathrm{FLAG}$ in strains carrying different CJnc180/190 promoter alleles. (+/-) indicates if a promoter is WT or mutant (see Fig 3C). (C \& D) The effect of CJnc180 overexpression or absence on ptmG. Levels of PtmG-3×FLAG protein were measured in the indicated strains in log phase (C) or at three growth phases (eExp, Exp, or Stat) (D) by western blot. Error bars represent the standard error of the mean. *: $\mathrm{p}<0.1,{ }^{* *}: \mathrm{p}<0.01,{ }^{* * *}: \mathrm{p}<0.0005,{ }^{* * * *}: \mathrm{p}<0.0005$, ns: not significant, Student's unpaired $t$-test $(\mathrm{n}=$ $3)$, vs. C-180/190 at the same growth phase. . For all northern blots, probes for the mature sRNAs (CSO-0189 and CSO-0185 for CJnc180 and CJnc190, respectively) and the 5' end of the ptmG ORF (CSO-1666) were used. As a loading control, 5S rRNA (CSO-0192) or RnpB (CSO-0497) was also probed. See also Fig S13. 